\title{
International fisheries agreements with a shifting stock*
}

\author{
Florian K Diekert ${ }^{\dagger} \quad$ Emmi Nieminen ${ }^{\ddagger}$
}

July 2015

\begin{abstract}
When a fish stock shifts from one nation to another nation, e.g. due to climate change, the nation that loses the resource has incentives to deplete it while the other nation, receiving the resource, has incentives to conserve it. We propose an analytical model to study under which circumstances self-enforcing agreements can align incentives. Our setup allows to distinguish between a fast and a slow shift in ownership and between a smooth or a sudden shift. We show that the shorter the expected duration of the shift, the higher the total equilibrium exploitation rate. Similarly, a sudden shift implies - by and large - more aggressive non-cooperative exploitation than a gradual transition. However, a self-enforcing agreement without side-payments is more likely for a sudden than for a smooth shift. Further, the scope for cooperation increases with the expected duration of the transition, and it decreases with the renewability of the resource and the discount rate. Most importantly, we show that concentrating on in-kind transfers can be very detrimental for shifting renewable resources: In some cases, no efficient bargaining solution exists, even when there are only two players.
\end{abstract}

Keywords: Renewable Resources; Dynamic Game; International Environmental Agreements; Regime Shift; Climate Change.

JEL Codes: C73, Q22, Q54

\section{Introduction}

Climate change will put existing International Fisheries Agreements under severe stress (Miller et al., 2013). In fact, this is already happening today. For example, the appearance of Atlantic mackerel in Icelandic waters has brought Iceland, Faroese, Norway and the EU

${ }^{*}$ This research is funded by NorMER, a Nordic Centre of Excellence for Research on Marine Ecosystems and Resources under Climate Change. We would like to thank Chris Costello, Rögnvaldur Hannesson, Marko Lindroos, and seminar participants at UCSB, the 2014 IIFET conference in Brisbane, and the 2014 Annual NorMER meeting in Copenhagen for their constructive comments and feedback. All remaining errors are, of course, our own.

${ }^{\dagger}$ Department of Economics and CEES, Department of Biosciences. University of Oslo, PO-Box 1095, Blindern, 3017 Oslo, Norway. E-mail: f.k.diekert@ibv.uio.no

${ }^{\ddagger}$ Department of Economics and Management. University of Helsinki, PO-Box 27, 00014 University of Helsinki, Finland. E-mail: emmi.e.nieminen@helsinki.fi 
to the brink of a new "fish war". The previous fisheries agreement has collapsed and the conflict over the level and distribution of fish quotas has - to date - not been resolved. Similarly, salmon stocks along the West Coast of Canada and the US have shifted their distribution depending on the climatic regime. This has repeatedly led to a break-down of the US-Canadian agreement on the Pacific salmon fisheries.

In this paper, we develop a dynamic non-cooperative game where the ownership of a productive resource shifts from one player to the other at an uncertain point in time. Contrary to the benchmark game of joint ownership but no shift, it is not always possible to achieve a stable, mutually beneficial, sharing agreement - even when there are only two players. A shifting distribution increases the bargaining position of the party whose share of the resource increases, while it decreases the bargaining strength of the other party. The first party thus wants to re-negotiate to obtain a more favorable agreement: The party receiving the resource stock will have more conservation incentives than the party losing the resource. Consider a pie which can be eaten exclusively by player $A$ in the first period, and whatever player $A$ leaves on the plate will be owned by player $B$ in the second period. Abstracting from discounting or uncertainty, player $A$ 's optimal strategy is to eat the entire pie in the first period. Without side-payments, there is no contract that player $B$ could offer player $A$ to make both strictly better off.

When the asset can be liquidated, or more generally transfers in utility are permitted, this tension is resolved immediately. The former party simply buys out the latter party. That is, player $B$ can just pay player $A$ the amount that is equivalent to eating half the pie in exchange for leaving half of it for the second period. In essence, the Coase-Theorem tells us that the allocation of initial property rights should not matter for the possibility to achieve an efficient bargaining solution. We show that this reasoning does not hold in a dynamic context when transfers are to be made in-kind.

Concentrating on in-kind transfers is particularly relevant for natural resources. For simple technological reasons, it may not be possible to liquidate fish stocks or forests in the same way as financial assets. More importantly, however, questions of national sovereignty, political legitimacy, and ethical concerns play an important role in this context. Quite generally, it is natural to restrict attention to in-kind transfers in a dynamic, intergenerational, setting. Future generations are simply not around to share the potential surplus from an efficient transfer of the resource with the current generation.

This is not to say that an analysis of financial side-payments is not interesting per se. Munro (1979) has pointed out more than 30 years ago that financial side-payments (or equivalently: direct profit-sharing) may greatly ease the prospects of attaining a cooperative solution - though the issue of time-consistency remains (Vislie, 1987). Relatedly, Costello and Kaffine (2011) show that unitization of a spatially connected renewable resource can yield the first-best outcome as a sub-game perfect equilibrium under profitsharing, but not under revenue-sharing. Indeed, profit sharing or financial side-payments are rarely observed in practice. Of course, the fact that one does not see a blunt statement such as "you receive $\mathrm{x}$ dollar for each ton of fish that I am allowed to harvest in your waters" does not mean that there are not more subtle ways of side-payments via issue- 
linkage in multi-layered international negotiations. Nevertheless, we argue that the scope of financial side-payments to enable international agreements is limited, especially when the utility derived from fishing captures broader aspects than just profits, such as cultural identity, tourism, employment, etc. It is politically, and to some extent also ethically, difficult to express these aspects in monetary terms.

We build on the "fish war" framework of Levhari and Mirman (1980) to develop a tractable model of an international fishery that is shared between two nations, and where the fish stock shifts from one player to the other. We solve for the feedback Nash-equilibrium of the dynamic non-cooperative game and characterize the scope of self-enforcing agreements to divide the optimal harvest share. Moreover, we explicitly consider different types of transition dynamics: Is non-cooperative exploitation more aggressive when the change in ownership occurs suddenly or gradually? Can cooperation more easily be achieved when the change in ownership is fast, or when it is slow?

\section{Relation to the literature}

The breakdown of the Coase Theorem in a dynamic setting without commitment is most prominently discussed in the literature on political economics (Dixit et al., 2000; Acemoglu, 2003). Recently, Harstad (2013) has applied these insights to environmental goods. In his setup, the owner, or the seller, of a "conservation good" (such as a tropical forest) desires to consume it, but the buyer benefits more from conservation. However, the buyer is also satisfied when the good is not consumed, and thus it has an existence value to the buyer. Contradictorily, the buyer is not willing to pay when the seller is conserving the good, and the seller is conserving only if the buyer is expected to buy, leading to the conclusion that a "market for conservation" is unlikely to exist.

Our paper adds to this by explicitly dealing with a dynamic renewable resource. It is thereby related to two strands of literature. First of all, it ties to the theoretical work on self-enforcing agreements over renewable resources. Using the Levhari-Mirman framework as we do, Kwon (2006) studies the existence of self-enforcing coalitions, finding - as it is common in the international agreements literature (Barrett, 1994) - that coalitions of more than two or three players are generally not stable. Using "farsighted" instead of Nash conjectures, Breton and Keoula (2012) derive less pessimistic results: Cooperation can be sustained under a much larger range of circumstances because threats of successive deviations are credible when players anticipate the formation of new coalition structures. Considering asymmetric players, however, does not yield similarly optimistic outcomes with respect to the size of stable coalitions (Breton and Keoula, 2014). Fesselmeyer and Santugini (2013) do not study coalition structures, but the exogenous risk of regime shifts in the "fish war" model, arguing that a negative shock may exacerbate the inefficiency implied by non-cooperation. Miller and Nkuiya (2014) then show that the threat of a negative regime shift may actually induce more cooperation and allow larger coalition sizes. We differ from the above contributions by considering a structurally asymmetric setup: The resource shifts from player $A$ to $B$, and while the shared ownership induces 
efficiency losses, it is the fact that $A$ loses the resource which hinders the formation of international agreements.

Therefore, our paper is also related to the literature on expropriation in the context of natural resources (Long, 1975; Ryszka, 2013). In our case, the rapacity effects of excessive resource depletion are not induced by exogenous expropriation risk, but by a combination of a regime shift risk with the presence of another strategic player.

Finally, we add to the growing body of research that specifically deals with the effects of climate change on international fisheries agreements. The first paper addressing in this line is from McKelvey et al. (2003), who argue that altered habitat or migration routes may destabilize existing agreements. The authors therefore argue for flexible quota shares. As we show here, flexible quota sharing certainly is important, but it may actually not be enough to avoid undesired competition. The model of McKelvey et al. (2003), which is an extension of the classical fish-war model of Levhari and Mirman (1980), is now known as the "split-stream model" and forms the basis of several papers: Liu et al. (2012) consider the distribution of the Norwegian spring-spawning herring and study partial coalition structures. They conclude that cost asymmetry improves the stability of the grand coalition, and non-cooperation gives more conservation incentives to the major player than the

minor player. Liu and Heino (2013) compare proactive management that takes possible climate change effects on the stock distribution into account with reactive management. They find that the players behave symmetrically under reactive management, but proactive management encourages the player losing the stock to act more aggressively. They conclude that the stock will be in greatest danger when both players own equal shares, confirming theoretical work of Hannesson (2007). Hannesson (2007) further points out that the transition of ownership may even lead to an extinction of the stock when harvesting costs are independent of the stock. The Northeast Atlantic mackerel stock is studied by Hannesson (2013) under three different hypothesis about what causes the shift in stock distribution. He concludes that the minor player has a stronger bargaining position when the migration pattern is deterministic or purely random. Under a stock-dependent migration, the major player has a threat strategy to fish down the stock to such a low level that it does not spill over to the minor player's zone. Another recent application to the same stock is Ellefsen (2013), confirming that the agreement is destabilized by a new entrant, and that it is the major player who has to pay most in order to encourage the new member to join. Because we go back to the "fish war" predecessor of the "split-stream model", we can derive explicit analytical solutions to the feedback equilibrium instead of relying on numerical simulations, or confining our discussion to steady-state outcomes.

\section{The Model}

Consider a situation with two neighbouring nations, $A$ and $B$, that derive utility from harvesting fish from a stock whose size at time $t$ is given by $x_{t}$. However, a nation's access to this resource may be limited, for example because the majority of the fish stock is outside the countries' exclusive economic zone (EEZ), or because one nation has specialized 
in harvesting a sub-population. Specifically, let $s_{t}$ be the share of the stock that country $A$ can access (due to technology or politics). Correspondingly, $1-s_{t}$ of the stock is "owned" by player $B$. Global warming (or some other form of environmental change) is expected to alter the distribution of the fish stock. Specifically, we will assume that, in any period, the players' share remains the same with probability $q$, and with probability $1-q$, there will be a shift in the distribution of the fish stock. We will generally presume that the stock moves from player $A$ to player $B$. That is, the share $s_{t}$, that player $A$ has access to, is expected to decline with time. Correspondingly, player $B$ expects to see an increase in the part of the resource that he can harvest.

There are several real-world cases that fit this generic description. For example, Atlantic Mackerel was basically absent from Icelandic waters and this fish stock was almost exclusively harvested by Norway and the European Union in the past. However, since 2006/07 more and more of the fish is found in Icelandic waters (Hannesson, 2013). It is yet to be seen whether stock shares will stabilize at the current levels, or whether the change continues so that the stock will eventually leave the Norwegian and European waters altogether. While access to the stock has a clear geographic/political interpretation in the former example, it could also have a technological interpretation. In the Baltic Sea, pelagic and demersal fisheries have always coexisted to some extent. Originally, the food web was dominated by cod, which was heavily fished. In the late 1980s, a rapid regime shift occurred and the system has since been dominated by small planktivorous fishes such as sprat (Casini et al., 2012). Recent research indicates that this change is likely to be permanent (Blenckner et al. 2015). When interpreting $x_{t}$ not as the stock of a specific species, but more broadly as the productive potential of an ecosystem, one can clearly say that the share of the resource $s$, that is available for fishing firms that specialize on catching cod, has declined dramatically, whereas the resource share has increased for those firms that have invested in gear to target small pelagics.

In this section, we will present the basic setup of our model and characterize the noncooperative equilibrium. In section 3, we discuss the scope for self-enforcing agreements, and in section 4, we explore differences in the quality of the shift (i.e. whether it is slow or fast, gradual or smooth).

\section{The basic setup}

We build on the classical model by Levhari and Mirman (1980) with two players that apply the same discount factor $\beta$. Time is discrete with $t=0,1,2, \ldots$ Players derive utility from consuming the resource according to $u\left(c_{t}^{i}\right)=\ln \left(c_{t}^{i}\right)$, where $i=A, B$. It is well known that this game has a unique Nash equilibrium in linear strategies (Long, 2010), and we will therefore concentrate on linear strategies. To simplify notation, we denote player $A$ 's extraction rate by $a_{t}$ and player $B$ 's extraction rate by $b_{t}$ (so that $c_{t}^{A}=a_{t} x_{t}$ and $c_{t}^{B}=b_{t} x_{t}$ ). Denote the total extraction rate by $d_{t}$ (so that $d_{t} \equiv a_{t}+b_{t}$ ). The resource 
develops according to:

$$
x_{t+1}=M\left(\left(1-d_{t}\right) x_{t}\right)^{\alpha}
$$

The parameter $\alpha$ determines the "renewability" of the resource with $\alpha \in(0,1)$ and the smaller is $\alpha$, the stronger is the natural re-growth potential of the resource. $M$ is a scaling parameter which has no effect on the optimal choice of the extraction rate. It ensures that the value-function of the players remains non-negative in all stages of the game $1^{1}$ For a constant exploitation rate $d$, the steady-state value of $x$ is given by $\bar{x}=M((1-d) M)^{\frac{\alpha}{1-\alpha}}$.

To allow for a general analysis of the effect of an anticipated, but uncertain shift in ownership, we assume that the transition of the resource from player $A$ to player $B$ proceeds in stages. In total, there are $T$ stages, and within each stage $\tau$ (where $\tau=$ $0,1, \ldots T)$ the share of the stock that player $A$ has access to is given by $s_{\tau}$ and the share of the stock that player $B$ has access to is given by $1-s_{\tau}$. In other words, the player's harvesting rates in a given stage $\tau$ are constrained by $a_{\tau} \leq s_{\tau}$ and $b_{\tau} \leq 1-s_{\tau}$. The duration of each stage is unknown, but there is a constant probability $q$ that the systems stays in the current stage and there is a constant probability $1-q$ that after any given period the next period starts in a new stage with $s_{\tau+1}$. We assume that $s_{0}=1$ and $s_{T}=0$. and we require that $s_{\tau}<s_{\tau+1}$. Other than that, we do not need to impose further restrictions on the way how the resource changes ownership. Note however, that the assumption that $s_{T}=0$ is not innocuous. It means that player $A$ completely loses her access to the resource, and that her time horizon is essentially finite (although it is uncertain when the end arrives).

It can be shown that when $s_{T}$ is larger than zero, no matter how small $s_{T}$ is, one would end up in a situation that is essentially the same as the standard Levhari-Mirman game. Structurally, the reason is that the marginal utility of consumption grows without bounds as $c \rightarrow 0$ when using a logarithmic utility function. We argue that this feature of the model does not have a meaningful interpretation in the case of real world fisheries. Rather, we understand our model so that when $s$ has dropped below some level, it does no longer pass the cost-benefit test to continue harvesting from this resource and the fishery is abandoned. (Recall that we scale the parameter $M$ so that the value of active harvesting is always positive).

\section{General pattern of the non-cooperative equilibrium}

For a given share of the resource, $s_{\tau}$, and a given size of the stock $x_{t}$, player $A$ (or player $B$ for that matter) maximizes utility from consuming a share of the resource now and in the future. The player takes into account that with probability $q$, the environmental regime will stay the same. Thus, the future value of the game, conditional on stock development, is the same as today. Denote this value by $V^{A}\left(s_{\tau}, x_{t}\right)$. With probability $1-q$, however,

\footnotetext{
${ }^{1}$ Let $i_{\min }$ be the lowest extraction rate of player $i$ and let $d_{\max }$ be the largest equilibrium total extraction rate. Then a condition that guarantees non-negative objective function values is $i_{\text {min }} M\left(\left(1-d_{\max }\right) M\right)^{\frac{\alpha}{1-\alpha}} \geq 1$ for $i=A, B$.
} 
the environmental regime will change to the next stage and the value of the game is then given by $V^{A}\left(s_{\tau+1}, x_{t+1}\right)$. In other words, there are two dynamic dimensions and two state variables in this game. Namely, (calendar) time $t$ and the index $\tau$ that counts which stage the game is in. The resource stock $x_{t}$ moves with calendar time, but the way the resource is shared (given by $s_{\tau}$ ) changes from stage to stage. Note that although the overall game is not stationary, the problem that the players face at any point in time is stationary.

Player $A$ 's and $B$ 's value functions are therefore given by:

$$
\begin{aligned}
& V^{A}\left(s_{\tau}, x_{t}\right)=\max _{a \leq s_{\tau}}\left\{\ln a x_{t}+\beta\left[q V^{A}\left(s_{\tau}, x_{t+1}\right)+(1-q) V^{A}\left(s_{\tau+1}, x_{t+1}\right)\right]\right\} \\
& V^{B}\left(s_{\tau}, x_{t}\right)=\max _{b \leq 1-s_{\tau}}\left\{\ln b x_{t}+\beta\left[q V^{B}\left(s_{\tau}, x_{t+1}\right)+(1-q) V^{B}\left(s_{\tau+1}, x_{t+1}\right)\right]\right\}
\end{aligned}
$$

As it is usual in "fish war" models, we suspect that also here, the player's value function is of the log-linear form $V^{i}\left(s_{\tau}, x_{t}\right)=k_{\tau}^{i} \ln x_{t}+K_{\tau}^{i}$ for $i=A, B$. This conjecture leads to the following first-order condition for player $A$ :

$$
\frac{1}{a_{\tau}}=\alpha \beta q k_{\tau}^{A} \frac{1}{1-a_{\tau}-b_{\tau}}+\alpha \beta(1-q) k_{\tau+1}^{A} \frac{1}{1-a_{\tau}-b_{\tau}}
$$

Accordingly, we can solve for player $A$ 's best-reply. Noting that her extraction can be limited by the accessible share $s_{\tau}$, we have:

$$
a_{\tau}=\min \left\{s_{\tau} ; \frac{\left(1-b_{\tau}\right)}{1+\alpha \beta\left(q k_{\tau}^{A}+(1-q) k_{\tau+1}^{A}\right)}\right\}
$$

The general case of player $B$ is symmetric, and hence his best-reply is:

$$
b_{\tau}=\min \left\{1-s_{\tau} ; \frac{\left(1-a_{\tau}\right)}{1+\alpha \beta\left(q k_{\tau}^{B}+(1-q) k_{\tau+1}^{B}\right)}\right\}
$$

We now set out to derive the structure of the coefficients $k_{\tau}^{i}$ and $K_{\tau}^{i}$. Noting that the structure is the same for player $A$ and $B$, we equate coefficients generically (where $j_{\tau}=a_{\tau}$ if $i=A$ and $j_{\tau}=b_{\tau}$ if $i=B$ ):

$$
\begin{aligned}
k_{\tau}^{i} \ln x+K_{\tau}^{i}= & \ln j_{\tau} x+\alpha \beta q\left[k_{\tau}^{i}\left(\ln \left(1-a_{\tau}-b_{\tau}\right) x+\frac{1}{\alpha} \ln M\right)+\frac{1}{\alpha} K_{\tau}^{i}\right] \\
& \quad+\alpha \beta(1-q)\left[k_{\tau+1}^{i}\left(\ln \left(1-a_{\tau}-b_{\tau}\right) x+\frac{1}{\alpha} \ln M\right)+\frac{1}{\alpha} K_{\tau+1}^{i}\right] \\
\Rightarrow & \\
k_{\tau}^{i}= & \frac{1}{1-\alpha \beta q}+\frac{\alpha \beta(1-q)}{1-\alpha \beta q} k_{\tau+1}^{i}
\end{aligned}
$$


This linear difference equation with constant coefficients can be solved ${ }^{2}$ to give:

$$
k_{\tau}^{i}=\left(\frac{\alpha \beta(1-q)}{1-\alpha \beta q}\right)^{T-\tau}\left[k_{T}^{i}-\frac{1}{1-\alpha \beta}\right]+\frac{1}{1-\alpha \beta}
$$

Note that the solution of $k_{\tau}^{i}$ will depend on $k_{T}^{i}$, which - in turn - depends on the utility derived from fishing in the last stage.

Player $A$ 's value of the fishery will be zero in the last stage $\left(s_{T}=0\right)$, so that we have $k_{T}^{A}=0$. This implies that $k_{\tau}^{A}=\frac{1}{1-\alpha \beta}\left(1-\left(\frac{\alpha \beta(1-q)}{1-\alpha \beta q}\right)^{T-\tau}\right)$. It is easy to see that $k_{\tau}^{A}>k_{\tau+1}^{A}$ : The term in the first bracket is smaller than one (as both $\alpha \beta<1$ and $q<1$ and therefore $\alpha \beta-\alpha \beta q<1-\alpha \beta q$ ), and the first term will therefore decrease as the exponent gets larger. In the beginning, $k_{\tau}^{A}$ will be close to the steady-state value of (7), $\frac{1}{1-\alpha \beta}$, especially when $T$ is large. For player $B$, we have $k_{\tau}^{B}=\frac{1}{1-\alpha \beta}$ for all $\tau$.

Although the coefficients $K_{\tau}^{A}$ and $K_{\tau}^{B}$ are irrelevant for the choice of the harvesting rate, we do need to spell them out in order to fully describe the value functions later on.

$$
K_{\tau}^{i}=\frac{1}{1-\beta q}\left[\ln j_{\tau}+\beta\left((1-q) K_{\tau+1}^{i}+\left(q k_{\tau}^{i}+(1-q) k_{\tau+1}^{i}\right)\left(\ln M+\alpha \ln \left(1-d_{\tau}\right)\right)\right]\right.
$$

Equation (8), in contrast to (6), is a difference equation where the coefficients are not constant, nor necessarily the same for both players. Solving it is therefore not instructive.

To describe the general pattern of the equilibrium in a concise form, we introduce the following auxiliary parameter:

$$
\gamma_{\tau}^{i} \equiv \frac{1}{1+\alpha \beta\left[q k_{\tau}^{i}+(1-q) k_{\tau+1}^{i}\right]} \quad \text { where } \gamma_{\tau}^{B}=\gamma^{B}=(1-\alpha \beta)
$$

Although the game is stationary within each stage, the extraction rates differ from stage to stage as summarized in the following proposition.

Proposition 1. The extraction pattern is characterized by at most three phases:

I. When $s_{\tau} \in\left[1,1-b_{\tau}\right)$, player B's extraction rate is constrained by the share of the resource available to him, while player $A$ 's rate is not. Total extraction rate $d_{\tau}$ is increasing with $\tau$ in Phase I. The individual extraction rates are given by:

$$
a_{\tau}=s_{\tau} \gamma_{\tau}^{A} \quad b_{\tau}=1-s_{\tau}
$$

II. When $s_{\tau} \in\left[1-b_{\tau}, a_{\tau}\right]$, neither player's extraction rate is constrained. Player A's extraction rate is increasing and player $B$ 's extraction rate is decreasing with $\tau$, and

\footnotetext{
${ }^{2}$ We refer to a standard mathematic textbook for economists e.g. Sydsæter et al. (2005, p.391). To see the solution more clearly, it is useful to change variables so that we start counting from the terminal stage $T$. That is, we introduce a new variable $n=0,1,2, \ldots$ so that $n=0 \equiv \tau=T$. This means that equation (6) is written as: $k_{n+1}^{i}=l+m k_{n}^{i}$ which can be solved to get $k_{n}^{i}=m^{n} k_{0}+l\left(\sum\left(m^{n}\right)\right)$. As we have a geometric series in the brackets, the solution of the equation can be rewritten as: $k_{n}^{i}=\frac{l\left(1-m^{n}\right)}{1-m}+m k_{0} \Leftrightarrow k_{n}^{i}=$ $m^{n}\left[k_{0}-\frac{l}{1-m}\right]+\frac{l}{1-m}$, where $\frac{l}{1-m}=\frac{1}{1-\alpha \beta q} \frac{1}{1-\frac{\alpha \beta(1-q)}{1-\alpha \beta q}}=\frac{1}{1-\alpha \beta q} \frac{1}{\frac{(1-\alpha \beta q)-\alpha \beta(1-q)}{1-\alpha \beta q}}=\frac{1}{1-\alpha \beta q-\alpha \beta+\alpha \beta q}=\frac{1}{1-\alpha \beta}$.
} 
the total extraction rate is increasing, where:

$$
a_{\tau}=\frac{\gamma_{\tau}^{A}\left(1-\gamma^{B}\right)}{1-\gamma_{\tau}^{A} \gamma^{B}} \quad b_{\tau}=\frac{\gamma^{B}\left(1-\gamma_{\tau}^{A}\right)}{1-\gamma_{\tau}^{A} \gamma^{B}}
$$

III. When $s_{\tau} \in\left(a_{\tau}, 0\right]$, the extraction rate of player $A$, but not player $B$ is constrained by the available share of the resource. Total extraction rate is decreasing with $\tau$.

$$
a_{\tau}=s_{\tau} \quad b_{\tau}=\left(1-s_{\tau}\right) \gamma^{B}
$$

Proof. The proof is given in Appendix A.1. Note that extraction is indeed linear in $x$ in all cases, so that the conjecture about the form of the value function is confirmed.

\section{Self-enforcing cooperative agreements}

In this section, we explore whether the players can agree on sharing the gains from maximizing the joint surplus of resource exploitation. It is well known that self-enforcing cooperative agreements of more than a few players are very difficult to form (Kwon, 2006; Breton and Keoula, 2014). Here we show that even two players may not be able to coordinate on the first-best if the stock shifts from one player to the other.

As we focus on in-kind transfers (ruling out financial side-payments), we ask whether the optimal total harvest can be split in such a way that each player's participation constraint - which is given by his payoff in absence of cooperation - is satisfied. In other words, we analyze whether there exists some share $\lambda$ so that player $A$ prefers obtaining that share of the socially optimal harvest rather than harvesting non-cooperatively, and whether player $B$ would at the same time prefer obtaining a share $(1-\lambda)$ rather than harvesting non-cooperatively. That is, we assume that at any time $t$, the players perceive the Nash equilibrium described above as the only alternative to a cooperative agreement for that stage. In essence, the players follow a grim trigger strategy within each stage. We do not require that the threat of non-cooperative play is renegotiation-proof. Such an agreement is self-enforcing in the sense that player $A$ has no incentive to harvest noncooperatively, given that player $B$ harvests cooperatively, and vice-versa.

To clearly distinguish the different continuation values, we denote the player's value function in the non-cooperative Nash equilibrium at stage $\tau$ by $V_{\tau}^{n c, i}\left(s_{\tau}, x_{t}\right)$. In contrast, $V_{\tau}^{c o o p, i}\left(\lambda, x_{t}\right)$ describes the value of cooperation for player $i$, given the share $\lambda$ and the current resource stock $x_{t}$. Let $V_{\tau}^{i} \equiv \max \left\{V_{\tau}^{c o o p, A}, V_{\tau}^{n c, A}\left(x_{t}\right)\right\}$, so that the function $V_{\tau+1}^{i}\left(x_{t+1}\right)$ is generic value of the game in the next stage (where it is a priori not known whether cooperative or non-cooperative play will dominate). Denoting the socially optimal total 
harvest rate by $d^{*}$, we can write:

$$
\begin{aligned}
& V_{\tau}^{\text {coop }, A}\left(\lambda, x_{t}\right)=\ln \left(\lambda d^{*} x_{t}\right)+\beta\left(q V_{\tau}^{\text {coop }, A}\left(\lambda, x_{t+1}\right)+(1-q) V_{\tau+1}^{A}\left(x_{t+1}\right)\right) \\
& V_{\tau}^{\text {coop }, B}\left(\lambda, x_{t}\right)=\ln \left((1-\lambda) d^{*} x_{t}\right)+\beta\left(q V_{\tau}^{\text {coop }, B}\left(\lambda, x_{t+1}\right)+(1-q) V_{\tau+1}^{B}\left(x_{t+1}\right)\right)
\end{aligned}
$$

Below, we show that reaching a cooperative agreement in any given stage $\tau$ does not depend on whether an agreement can be reached in the next stage. Changes in the external conditions may, in accordance with public international law 3 , make the current treaty inapplicable and will in fact often lead to re-negotiations of existing agreements. Therefore, in any given stage $\tau$, a cooperative agreement can be reached for that stage if condition (13) holds for any value of $x_{t}$.

$$
V_{\tau}^{\text {coop }, A}\left(\lambda, x_{t}\right) \geq V_{\tau}^{n c, A}\left(x_{t}\right) \quad \text { and } \quad V_{\tau}^{\text {coop }, B}\left(\lambda, x_{t}\right) \geq V_{\tau}^{n c, B}\left(x_{t}\right)
$$

Clearly, $V_{\tau}^{\text {coop }, A}\left(\lambda, x_{t}\right)$ is increasing in $\lambda$ and $V_{\tau}^{\text {coop }, B}\left(\lambda, x_{t}\right)$ is decreasing in $\lambda$. Let therefore $\lambda_{\text {min }}^{A} \in(0,1]$ be the minimum share of the optimal harvest that player A would be willing to accept in order to approve cooperative management. Similarly, let $\lambda_{\max }^{B} \in(0,1]$ the maximum share that player B would be willing to give to player A and still harvest cooperatively. Accordingly, we can define the scope for cooperation by:

$$
\Gamma \equiv\left\{\lambda_{\max }^{B}-\lambda_{\min }^{A} ; 0\right\}
$$

This allows us to state the following proposition:

Proposition 2. Cooperation possibilities are not constrained by the available harvest shares, but the scope for cooperation vanishes as $q \rightarrow 0$ or $\alpha \beta \rightarrow 1$. If there is scope for cooperation $(\Gamma>0), \lambda_{\min }^{A}$ and $\lambda_{\max }^{B}$ are given by equation (15) and (16), respectively (where $\varphi_{\tau}^{A}=q k_{\tau}^{A}+(1-q) k_{\tau+1}^{A}$ and $\left.\varphi^{B}=\frac{1}{1-\alpha \beta}\right)$.

$$
\begin{aligned}
\lambda_{\min }^{A} & =\frac{a_{\tau}}{1-\alpha \beta}\left(\frac{1-a_{\tau}-b_{\tau}}{\alpha \beta}\right)^{\alpha \beta \varphi_{\tau}^{A}} \\
\lambda_{\max }^{B} & =1-\frac{b_{\tau}}{1-\alpha \beta}\left(\frac{1-a_{\tau}-b_{\tau}}{\alpha \beta}\right)^{\alpha \beta \varphi^{B}}
\end{aligned}
$$

Proof. The proof is given in Appendix A.2.

Intuition can be built by considering the following simple example, which effectively sets $q=0$ and $\alpha \beta=1$ : There are only two periods, the resource is completely nonrenewable, and player $A$, who owns the resource entirely in the first period, will for sure

\footnotetext{
${ }^{3}$ The legal principle that treaties must be honoured (pacta sunt servanda) is understood to be contingent on the current state of affairs when the treaty was made (rebus sic stantibus).
} 
lose it in the next period. Even without discounting, player $A$ has no incentive to leave anything for player $B$. In fact, as $A$ 's best-reply is to consume the entire resource, there is no share that player $B$ can offer player $A$ to make both strictly better off.

More generally, it can be seen from equations (15) and (16) that the scope for cooperation depends in a complicated way on $\alpha, \beta$ and $q$. We therefore illustrate how the scope for collaboration depends on these parameters in Figure 1. We plot a grid of 100x100 combinations of $\alpha \beta$ between 0.5 and 0.9 (on the x-axis) and $q$ between 0.5 and 0.9 (on the $y$-axis) and check for each combination whether $\Gamma>0$ in all stages. If yes, we color that box green, if not, we color it red. We do so for three different values of $T$. The pattern that emerges is that the scope for cooperation is a little larger when $T=5$ rather than $T=15$, but these changes are small compared to the detrimental impact of increasing $\alpha \beta$ or decreasing $q$. This means that the scope of cooperation is larger the slower the shift, and - maybe counterintuitively - the less gradual the shift. The next section will deal with these qualitative differences of the transition dynamics in more detail.
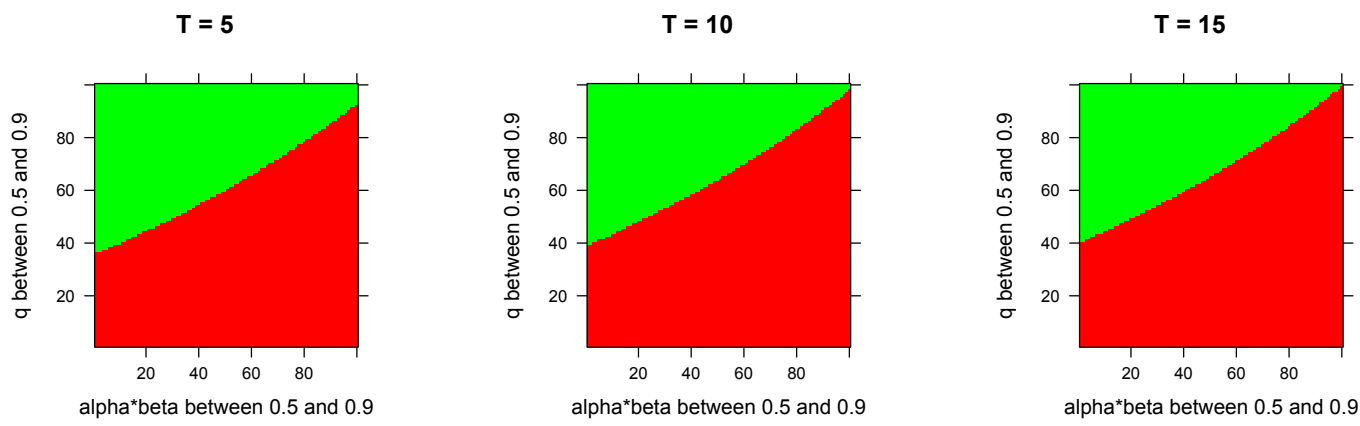

Figure 1: Parameter values for which it is possible to have cooperation in all stages.

\section{Comparative statics}

In this section, we discuss more specifically how the non-cooperative extraction pattern develops over the different stages of the game and how that in turn depends on the various parameters of the model.

But before that, it will be useful to introduce another variable: Denote by $Z$ the expected value of the time that it takes for the transition of the resource from player $A$ to player $B$ to complete. When $Z$ is small, we talk of a fast shift, and when $Z$ is larger, we talk of a slow shift. Now for a given $Z$, we could have a sudden shift, when there are few different stages, but $q$ is large so that the process - on average - stays a long time in each stage. We call such a transition dynamic abrupt. We talk of a gradual transition when, for a given $Z$, the change is characterized by many small steps. That is, $T$ is large and $q$ is relatively small. As the duration of each stage $\tau$ follows a geometric distribution with mean $\frac{1}{1-q}$, the total expected time that it takes until player $\mathrm{B}$ is the sole-owner is given by: $Z=\frac{T}{1-q}$.

In order to plot how the non-cooperative extraction rate and the scope for cooperation 
change over the transition phase, and to analyze how this depends on $Z, q$, and $T$, we impose the following structure on $s_{\tau}$ :

$$
s_{\tau}=\frac{T-\tau}{T}
$$

Figure 2 illustrates the non-cooperative extraction rates in equilibrium (where Player $A$ is red and Player $B$ is green). We plot three scenarios with the same expected length of the transition $(Z=10)$, but different values of $q$ and $T$. Note that the first and last stage are the situations where player $A$ or player $B$ are the sole-owner, respectively. Consequently, the harvest rate of the respective opponent is zero for these stages.
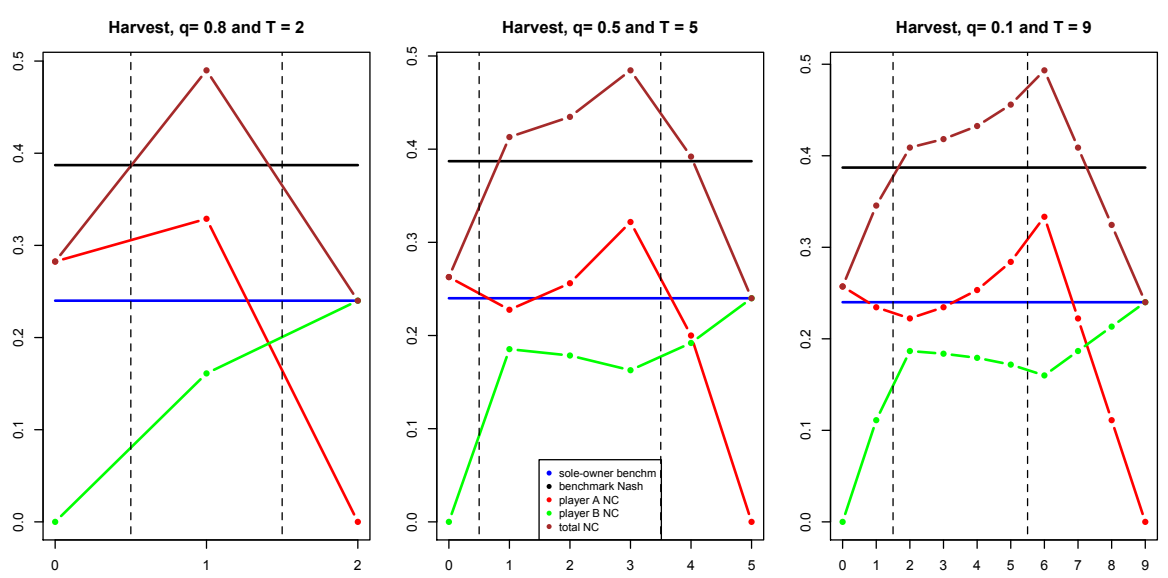

Figure 2: Extraction rates over the different stages of the game. $\alpha=0.8, \beta=0.95, M=3$

When the change in ownership is abrupt $(T=2)$, each phase consists of exactly one stage. (Phases are indicated by the dashed vertical lines.) When the change in ownership is more gradual ( $T=5$ and $T=9$ ), the duration of the phases expands and the relative changes in the individual harvest rates become visible. In particular in Phase II, when neither player is constrained, player $B$ 's extraction rate is decreasing in anticipation that he will eventually become the sole-owner of the resource. However, player $A$ 's extraction rate is increasing so strongly that the total extraction rate is increasing, too.

It follows immediately from Proposition 1 that the harvest rate reaches its highest value at the last stage of Phase II or at the first stage of Phase III. This stands in contrast to the steady state analysis of Hannesson (2007), where the stock is in the greatest danger of being extinct when the players share the stock equally. In fact, the stock will never be extinct here because utility is stock-dependent in our model. The total harvest rate is increasing throughout Phase II because player $A$ 's incentives to over-harvest are increasing the less access she has to the stock, while player $B$ 's conservation incentives are not strong enough to counteract that ${ }^{4}$

To further explore the interactions between a fast/slow versus a sudden/gradual shift,

\footnotetext{
${ }^{4}$ Although Liu and Heino do not discuss this issue explicitly, a close inspection of the figures in Liu and Heino (2013) suggests that the same result may also apply in their model.
} 
we plot the maximum harvest rate, $d_{\max }$, over the transition for different values of $Z, q$ and $T$. The blue squares in Figure 3 show $d_{\max }$ when we keep $Z$ fixed at a value of 10 years and $T$ increases by integer increments. The black circles show $d_{\max }$ when we keep $Z$ fixed at a value of 20 and the grey triangles show $d_{\max }$ when we keep $Z$ fixed at a value of 30 . First of all, we observe that $d_{\max }$ decreases with a slower change (a longer expected time of transition, i.e. a higher value of $Z$ ). Second, we note that for a given $Z$, a more gradual change (higher $T$ and lower $q$ for a given $Z$ ) means a lower $d_{\max }$ - on average. The trend for the grey, black, and blue points in Figure 3 is negative, but there is a considerable amount of variation. The jumps in $d_{\max }$ are caused by the discrete nature of the different stages.5

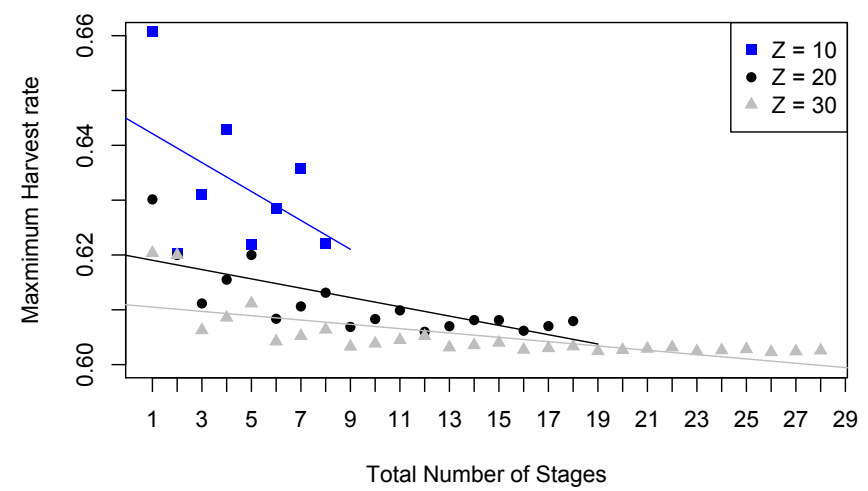

Figure 3: Maximum harvest rate as a function of $T$ for three different values of $Z$

Proposition 3. An faster shift (a decrease in Z) implies higher non-cooperative harvesting pressure. A more abrupt shift (increasing $q$ while decreasing $T$ for a given value of $Z$ ) has an ambiguous effect on the total extraction rate: A larger $q$ decreases total extraction pressure in phase I and II, but has no effect in phase III. A smaller T decreases total extraction in phase I and II, but increases extraction pressure in the last phase.

Proof. The proof is given in Appendix A.3.

Consider a reduction in $Z$, the expected time of transition. If this reduction comes about by decreasing the probability that the system stays in a given period, but keeping $T$ (the total number of stages) fixed, it will have no effect on $\gamma^{B}$. However, upon inspection of equation (7) and (9), one can see that $q$ enters both in the expression of $\gamma_{\tau}^{A}$ directly and indirectly via the expression of $k_{\tau}^{A}$. Thus, the main effect here really comes from the fact that player $A$ 's future value in any given period is depressed. Much like an increased discount rate, this induces her to be more rapacious. In contrast, if the reduction of the expected transition time comes about by reducing the number of stages $T$, while keeping $q$ fixed, player $A$ 's future is also depressed, but this channel only works by reducing $k_{\tau}^{A}$.

\footnotetext{
${ }^{5}$ In a sense, the lower $Z$, the stronger is the effect of an increase in $T$ on $q$. For example if $Z=3, T$ can only take the values 1,2 , and 3 . If $T=1, q=\frac{2}{3}$ and $d_{\max }=0.49$. When $T=2, q=\frac{1}{3}$ and $d_{\max }=0.62$ and when $T=3, q=0$ and $d_{\max }=0.62$. Now when $Z=50, q=0.3$ when $T=35$ and $q=0.28$ when $T=36$. The associated change in $d_{\max }$ will be much smaller.
} 
In addition, however, a change in $T$ also affects player $B$ 's strategy: A lower $T$ means that the scope for player $B$ to counteract $A$ 's overharvesting is decreased, especially in Phase III of the game. In that phase, player $A$ harvests all that is accessible to him, and a lower $T$ means that $s_{\tau}$, and hence player $A$ 's harvest, is higher.

An additional measure of particular interest is the lowest biomass that the stock will most likely obtain over the transition from player $A$ to player $B$. It is of crucial importance when there are potential non-linear effects at low abundance. (We do not explicitly consider concepts such as Allee effects or minimum viable population sizes here, but they could of course play a critical role in the real world.) Therefore, we analyze the size of the expected minimum stock biomass, $x_{\min }$ for different values of $q$ and $T$ (Table 1 ). The calculation of the expected minimum stock biomass, $x_{\min }$ takes into account that the expected length that the game stays in a given period $\tau$ will affect the expected value of the biomass at the beginning of the next stage. We assume that the game starts at the equilibrium value $x_{0}=M\left(\left(1-a_{0}\right) M\right)^{\frac{\alpha}{1-\alpha}}$. To make a concrete example, suppose $x_{0}=10$, total firststage equilibrium extraction $d_{1}=0.4$ and $\alpha=0.8, M=3$. When $q=0.5$, the expected time that the game stays in one stage is given by $\frac{1}{1-q}=2$ years. The expected value at the beginning of stage 2 is therefore given by: $x_{2}=M\left(\left(1-d_{1}\right)\left[M\left(\left(1-d_{1}\right) x_{0}\right)^{\alpha}\right]\right)^{\alpha}=15.1$. In contrast, when $q=0.9$, the game stays, on average, 10 years in a given stage, so that the value of $x$ at the onset of stage 2 would be $x_{2}=26.9$, which is a lot closer to the equilibrium value $M\left(\left(1-d_{1}\right) M\right)^{\frac{\alpha}{1-\alpha}}=31.5$.

Table 1: Expected minimum biomass and maximum extraction rate over the transition, $q$ is probability of staying in stage $s_{\tau} ; T$ is number of steps. Parameters: $\alpha=0.6 ; \beta=0.95$.

\begin{tabular}{cccc}
\hline & $T=2$ & $T=5$ & $T=9$ \\
\hline \hline$q=0.1$ & - & - & $Z=10$ years \\
& & & $x_{\min }=31.2$ \\
& & & $d_{\max }=0.49$ \\
\hline \multirow{4}{*}{$q=0.5$} & $Z=4$ years & $Z=10$ years & $Z=18$ years \\
& $x_{\min }=23.7$ & $x_{\min }=30.1$ & $x_{\min }=30.6$ \\
& $d_{\max }=0.62$ & $d_{\max }=0.48$ & $d_{\max }=0.43$ \\
\hline \multirow{4}{*}{$q=0.8$} & $Z=10$ years & & $Z=45$ years \\
& $x_{\min }=28.8$ & - & $x_{\min }=30.9$ \\
& $d_{\max }=0.49$ & & $d_{\max }=0.41$ \\
\hline
\end{tabular}

Table 1 confirms that the slower the change, the less pressure the stock is exposed to. Consider the second row (where $q$ is kept fixed and $T$ increased): As the expected duration of the transition increases from $Z=4$ years to $Z=18$ years, the maximal harvest rate declines from 0.62 to 0.43 . Similarly, the expected minimal value of the stock biomass increases from 23.7 to 30.6 . While the decline in maximal harvest rate is monotone, this is not the case for $x_{m i n}$ : Inspection of the third column reveals that $x_{m i n}$ actually decreases from a value of 31.2 to a value of 30.6 when $Z$ increases from 10 to 18 years by keeping 
$T$ fixed but increasing $q$. This non-monotonicity is driven by the fact that at $q=0.1$ the game stays in a given stage for only a year on average, so that the biomass at the expected time of transition is heavily influenced by the initial values. In contrast, the stock is expected to stay in a given stage much longer when $q=0.5$, so that the malign effect of non-cooperation plays out longer and essentially swamps the beneficial effect of a slower change.

Similarly, as discussed in Proposition 3 above, Table 1 shows how the effect of a more gradual or more sudden change is not monotonic. Consider the diagonal where $Z=10$ years for all entries. As we move from the bottom-left to the top-right, the change becomes much more gradual. However, the maximal harvest rate first decreases and then increases. In this case, the minimal biomass actually increases steadily as the change becomes smoother.

Finally, we consider the effect of a change in the resource's renewability, $\alpha$, and in the discount rate $\beta$. Note that $\alpha$ and $\beta$ always enter multiplicative as $\alpha \beta$ in $k_{\tau}^{i}$ and $\gamma_{\tau}^{i}$. Hence, in much of the literature, $\alpha \beta$ is treated as one parameter, the "bionomic discount factor".

Proposition 4. An increase in the bioeconomic parameters $\alpha$ and $\beta$ leads to a lower total extraction rate in all phases.

Proof. The proof is given in Appendix A.4.

An increase in $\alpha$ means the natural regrowth potential of the resource becomes weaker, inducing the players to harvest less aggressively. By comparing $110-(12)$ it becomes clear, that this effect is strongest in the third phase, when player $A$ 's harvesting is already constrained by the available share of the resource, and weakest in the second phase when neither player's harvesting is constrained.

An increase in the discount factor, $\beta$, means that the players place a higher value on the future harvesting opportunities. Therefore, a higher discount factor naturally leads to a decreased extraction rate. Again, this effect is weakest in the second phase.

\section{Gains from cooperation}

When illustrating under which circumstances a cooperative solution could be sustained by a self-enforcing agreement (Figure 1), we only considered whether there was any stage in which there would be no scope for collaboration. To obtain a better insight into how the dynamics unfold over the course of the transition, we show how the bargaining set changes over the different stages of the game in Figure 4. In particular, it becomes obvious how the scope for collaboration is compressed at the end and the beginning of the transition phase. This is not surprising, as it is here that both players have the strongest bargaining positions. Towards the end, for example, player $A$ has nothing left to lose, but also player $B$ has little incentive to give him much as player $A$ 's harvest share is constrained anyway. Furthermore, Figure 4 illustrates how player $A$ 's bargaining strength is declining over the different stages, though not in a monotonic fashion. Indeed, the changes from one stage to the next can be quite large, especially in the beginning or the end. 

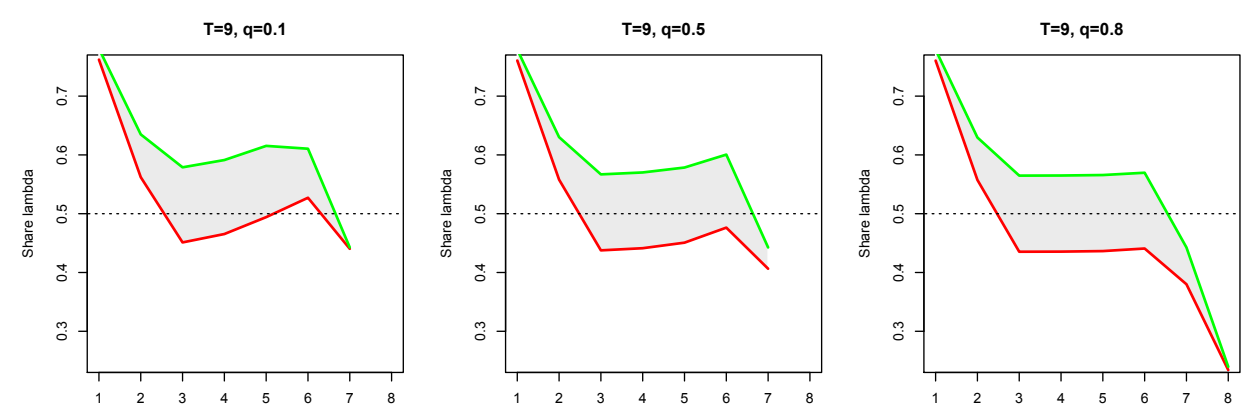

Figure 4: Illustration of bargaining set at different stages, $\alpha=0.6, \beta=0.95$

In order to speak about the potential gains from cooperation, we need to define the social optimum. We take the stance that the first-best is achieved when the sum of player's utility is maximized. Note that this implies that the shift of the fish stock is in fact immaterial. No matter who has access to what fraction of the stock, it will always be optimal to maximize the return the resource. That is, one can disregard the transition dynamics and simply write the social planner's problem as choosing an extraction pattern $d$ and then splitting it in some arbitrary way. For simplicity, we presume that the social planner does not discriminate between player $A$ and player $B$, so that $a^{*}=\frac{1}{2} d^{*}$ and $b^{*}=\frac{1}{2} d^{*}$. Thus, we can formulate the problem as finding the first-best total harvest as:

$$
V^{*}(x)=\max _{d \leq 1}\left\{2 \ln d x+\beta V^{*}\left(M((1-d) x)^{\alpha}\right)\right\}
$$

As usual we can use the 'guess and verify' method to solve for the value function. This confirms that $V^{*}=k^{*} \ln x+K^{*}$, where:

$$
k^{*}=\frac{1}{1-\alpha \beta} ; \quad K^{*}=\frac{1}{1-\beta}\left[\frac{\alpha \beta \ln \alpha \beta}{1-\alpha \beta}+\ln (1-\alpha \beta)+\frac{\beta \ln M}{1-\alpha \beta}-\ln 2\right]
$$

Consequently, the first-best total extraction and the optimal steady state resource stock are given by:

$$
d^{*}=(1-\alpha \beta) ; \quad \bar{x}^{*}=M(\alpha \beta M)^{\frac{\alpha}{1-\alpha}}
$$

In Figure 5, we then illustrate the development of the gains from cooperation. Obviously, the first-best value of the resource will not change with the transition of ownership, while player $A$ 's outside option (his non-cooperative value function $V^{A}\left(s_{\tau}, x_{t}\right)$, is given by equation (2) and plotted in red) decreases. Contrarily, player $B$ 's outside option increases. Overall one can see that player $A$ 's value function declines faster than player $B$ gains in value, so that the loss induced by non-cooperation is increasing with $\tau$. Or stated the other way around, the gains from cooperation are higher towards the end of the transition period. However, in accordance with Figure 4, one can see that it is also here that the cooperation is most difficult to achieve. Indeed, for our example, self-enforcing agreements 
cannot be formed in the last stage of the game when $T=9, q=0.1$ and $T=9, q=0.5$. Furthermore, one can see that player $A$ 's value function is higher, the higher $q$ (but note the overall loss from non-cooperation is not very sensitive to $q$ ). This is simply a consequence of the model setup where the resource shifts entirely from player $A$ to player $B$. In other words, the larger is $q$, the shorter is $A$ 's expected time horizon.
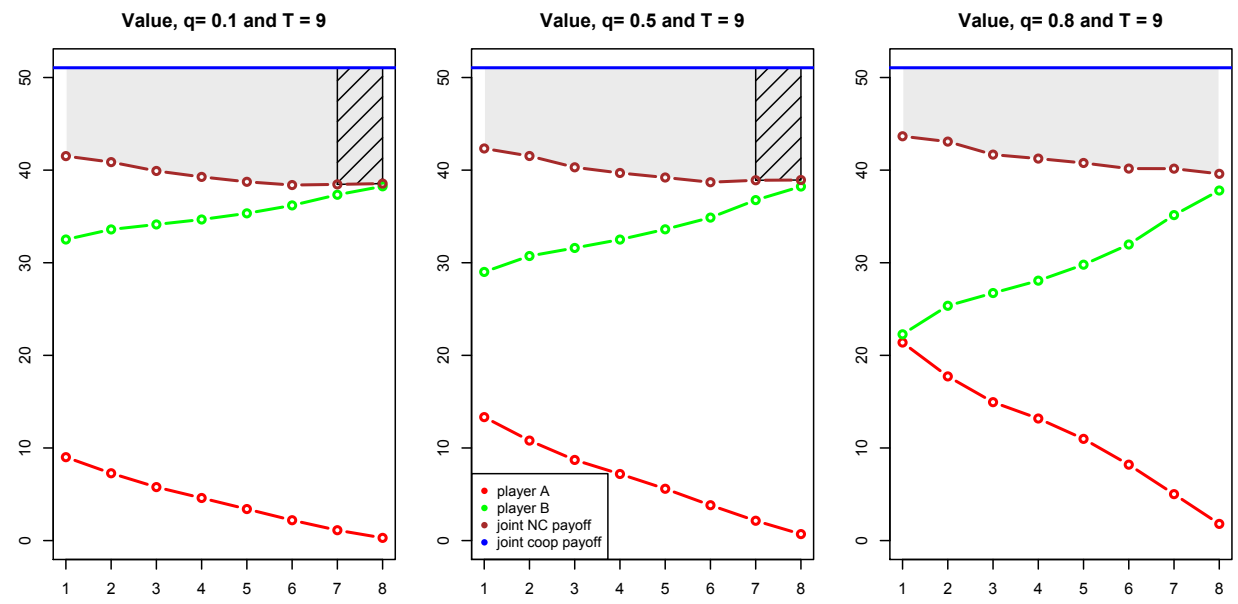

Figure 5: Development of the non-cooperative value $($ red $=$ player $A$, green $=$ player $B$ ) and the gains from cooperation (gray area, hatched when self-enforcing agreement not attainable). $\alpha=0.8, \beta=0.95, M=3$

\section{Discussion and Conclusion}

We extend the well-known model of Levhari and Mirman (1980) to study the situation where a renewable resource changes the hand of its owner in an anticipated way, but at an uncertain time. We show that the shorter the expected duration of the transition (the faster the shift occurs), the higher the total extraction rate. There are really two different effects at play: First, a faster shift could mean that the probability to stay in the current regime is reduced. This decreases the future value of the game for player $A$ (who loses the resource) and hence increases her incentives to over-harvest. Second, the expected duration of the shift could be reduced by decreasing $T$, which makes the shift more abrupt. This also decreases player $A$ 's future value, but it also means that player $B$ can do less to counteract the aggressiveness of player $A$ because he has less leverage. In addition to disentangling the effect of a slow or fast change from the effects of a gradual or sudden change, we point out that a decrease in the player's valuation of the future and an increase in the renewability of the resource leads to a higher total extraction rate.

Furthermore, we study the conditions under which the players can sustain self-enforcing agreements to share the optimal harvest level. We show that, in contrast to the benchmark game of no shift and in contrast to the case with side-payments, even two players cannot always form an agreement. As in non-cooperative equilibrium, the slower the shift is, the better are the chances to achieve cooperation. However, the scope for cooperation 
decreases as the change is more gradual. The scope for cooperation vanishes when the regrowth potential of the resource is low simultaneously with a high discount factor.

Although our analytic model is fairly general, there are of course a number of (implicit) simplifying assumptions that warrant discussion: First, we have assumed the regime shift to be irreversible. Though clearly a simplifying assumption, it is not entirely clear what additional insights the alternative assumption would yield. Certainly, the nature of the game would change if the stock shifted back to the original owner at some point. Then the player losing the stock would have more conservation incentives as it has the chance to continue harvesting in the future. On the other hand, the player receiving the stock would also take this into consideration and not harvest as a sole-owner, as there is a probability to lose the stock to the original owner. This implies that the asymmetric game of the stock shifting from one to the other player is embedded in a larger symmetric game. In the extreme case, this larger symmetric game would converge to the benchmark game without a shift in stock distribution.

Second, we have concentrated on the case with two players only. In general, the literature shows the difficulty to sustain a cooperation between multiple players, especially when no additional enforcement mechanism are available to alleviate the problem. Breton and Keoula (2014) showed that an assumption of asymmetric players is not sufficient to facilitate a coalition of several players, and the largest coalition size in their model is two. In our case, the cooperation is not always possible even with two players, and if we added a third (or several) players into the game, the possibility to achieve an agreement may vanish entirely. However, in a recent contribution Miller and Nkuiya (2014) show that coalition can be larger under threat of regime shift. In essence we have modeled the transition in stock distribution as a series of exogenous regime shifts. Hence it would be a very interesting avenue for future research to explore how the game changes when we allow for more than two players. It goes without saying that this would require many more structural assumptions on how the changes in the resource distribution affect the share of the stock that the players have access to.

Finally, we have assumed that both the harvest of the players and the resource stock is perfectly observable and common knowledge. Uncertainty about the resource stock has no effect on the non-cooperative extraction decisions in the Levhari-Mirman game, as shown by Antoniadou et al. (2013). When the level of individual extraction is unobservable to society, a moral hazard problem arises, as it becomes impossible to identify the cheater and thus target the punishment correctly. Therefore, Tarui et al. (2008) suggests a symmetric punishment for all agents. They introduce a two-phase punishment scheme, in which a subgame perfect equilibrium with the lowest possible payoffs is brought into play in the first phase followed by a second phase with a forgiveness from all agents and furthermore a stock re-establishment to the cooperative level. In line with our results, Tarui et al. (2008) point out that a small number of players, fast growth rate of the resource or high discount factors may not support cooperation when actions are unobservable. An alternative to symmetric punishment could be the random punishment introduced scheme by Jensen and Kronbak (2009), or the scheme suggested by Laukkanen (2003), according to which 
cooperation is enforced by the threat of a sufficiently detrimental non-cooperative Nash equilibrium. This scheme provides cooperation, but under a narrower set of conditions than the one suggested in Tarui et al. (2008). In short, these studies indicate that including uncertainty about the other player's harvest (in combination with the risk-aversion implied by a concave utility function) should lead to wider range under which cooperation is sustainable, possibly even when there are more than two players. Whether this conjecture indeed holds is an interesting avenue for further research.

Our model speaks to the recent difficulties that existing international fisheries agreements have encountered as fish stocks have changed their spatial distribution. These difficulties are indeed expected to increase due to climate change. A clear policy recommendation that follows from our analysis is to focus attention on those cases where the changes are expected to occur fast and smoothly. In particular, mechanisms to enlarge the bargaining space of international fisheries by stock-unrelated side-payments should be investigated for those cases. 


\section{References}

Acemoglu, D. (2003). Why not a political Coase theorem? Social conflict, commitment, and politics. Journal of Comparative Economics, 31(4):620-652.

Antoniadou, E., Koulovatianos, C., and Mirman, L. J. (2013). Strategic exploitation of a common-property resource under uncertainty. Journal of Environmental Economics and Management, 65(1):28-39.

Barrett, S. (1994). Self-enforcing international environmental agreements. Oxford Economic Papers, 46(0):878-94.

Blenckner, T., Llope, M., Möllmann, C., Voss, R., Quaas, M. F., Casini, M., Lindegren, M., Folke, C., and Chr. Stenseth, N. (2015). Climate and fishing steer ecosystem regeneration to uncertain economic futures. Proceedings of the Royal Society of London B: Biological Sciences, 282(1803).

Breton, M. and Keoula, M. Y. (2012). Farsightedness in a coalitional great fish war. Environmental and Resource Economics, 51(2):297-315.

Breton, M. and Keoula, M. Y. (2014). A great fish war model with asymmetric players. Ecological Economics, 97:209-223.

Casini, M., Blenckner, T., Möllmann, C., Gårdmark, A., Lindegren, M., Llope, M., Kornilovs, G., Plikshs, M., and Stenseth, N. C. (2012). Predator transitory spillover induces trophic cascades in ecological sinks. Proceedings of the National Academy of Sciences, 109(21):8185-8189.

Costello, C. and Kaffine, D. (2011). Unitization of spatially connected renewable resources. The B.E. Journal of Economic Analysis and Policy, 11(1):1-29.

Dixit, A., Grossman, G., and Gul, F. (2000). The dynamics of political compromise. Journal of Political Economy, 108(3):531-568.

Ellefsen, H. (2013). The stability of fishing agreements with entry: The northeast atlantic mackerel. Strategic Behavior and the Environment, 3(1-2):67-95.

Fesselmeyer, E. and Santugini, M. (2013). Strategic exploitation of a common resource under environmental risk. Journal of Economic Dynamics and Control, 37(1):125-136.

Hannesson, R. (2007). Global warming and fish migration. Natural Resource Modeling, 20(2):301-319.

Hannesson, R. (2013). Sharing a migrating fish stock. Marine Resource Economics, 28(1):1-17.

Harstad, B. (2013). The market for conservation and other hostages. CESifo Working Paper Series 4296, CESifo Group Munich.

Jensen, F. and Kronbak, L. G. (2009). Random penalties and renewable resources: a mechanism to reach optimal landings in fisheries. Natural Resource Modeling, 22(3):393-414.

Kwon, O. S. (2006). Partial international coordination in the great fish war. Environmental and Resource Economics, $33(4): 463-483$.

Laukkanen, M. (2003). Cooperative and non-cooperative harvesting in a stochastic sequential fishery. Journal of Environmental Economics and Management, 45(2, Supplement):454 - 473.

Levhari, D. and Mirman, L. J. (1980). The Great Fish War: An Example Using a Dynamic Cournot-Nash Solution. The Bell Journal of Economics, 11(1):322-334.

Liu, X. and Heino, M. (2013). Comparing proactive and reactive management: Managing a transboundary fish stock under changing environment. Natural Resource Modeling, forthcoming:no-no.

Liu, X., Lindroos, M., and Sandal, L. (2012). Sharing a fish stock with density-dependent distribution and unit harvest costs. In 14th International BIOECON Conference, Cambridge, UK.

Long, N. V. (1975). Resource extraction under the uncertainty about possible nationalization. Journal of Economic Theory, 10(1):42-53.

Long, N. V. (2010). A Survey of Dynamic Games in Economics, volume 1 of Surveys on Theories in Economics and Business Administration. World Scientific, Singapore.

McKelvey, R., Miller, K., and Golubtsov, P. (2003). Fish wars revisited: a stochastic incomplete-information harvesting game. In Wesseler, J., Weikard, H.-P., and Weaver, R. D., editors, Risk and Uncertainty in Environmental and Natural Resource Economics, pages 93-112. Edward Elgar.

Miller, K. A., Munro, G. R., Sumaila, U. R., and Cheung, W. W. L. (2013). Governing marine fisheries in a changing climate: A game-theoretic perspective. Canadian Journal of Agricultural Economics, 61(2):309-334.

Miller, S. and Nkuiya, B. (2014). Coalition formation in fisheries with potential regime shift. Unpublished Working Paper, University of California, Santa Barbara.

Munro, G. R. (1979). The Optimal Management of Transboundary Renewable Resources. The Canadian Journal of Economics / Revue canadienne d'Economique, 12(3):355-376.

Ryszka, K. (2013). Resource extraction in a political economy framework. Discussion Paper TI 13-094/VIII, Tinbergen Institute.

Sydsæter, K., Hammond, P., Seierstad, A., and Strøm, A. (2005). Further Mathematics for Economic Analysis. Prentice Hall, Harlow.

Tarui, N., Mason, C. F., Polasky, S., and Ellis, G. (2008). Cooperation in the commons with unobservable actions. Journal of Environmental Economics and Management, 55(1):37 - 51.

Vislie, J. (1987). On the Optimal Management of Transboundary Renewable Resources: A Comment on Munro's Paper. The Canadian Journal of Economics / Revue canadienne d'Economique, 20(4):870-875. 


\section{Appendix}

\section{A.1 Proof of Proposition 1}

The existence of the first phase follows from the assumption that $s_{0}=1$ so that player $\mathrm{A}$ is the sole-owner in the first stage of the game, and player B cannot extract anything. The existence of the last phase follows from the assumption that $s_{T}=0$ and player A cannot extract anything. The second phase may not exist (e.g. when $T=1$ ).

Consider the first phase (equation 10p. Player B's extraction rate is constrained by the share $1-s_{\tau}$ accessible to him. This share is increasing as $s_{\tau}$ is decreases. The extraction rate of player A is given by $a_{\tau}=s_{\tau} \gamma_{\tau}^{A}$. This may in principle increase or decrease. Clearly, $s_{\tau}$ is declining with $\tau$, but $\gamma_{\tau}^{A}$ increases with $\tau$ :

$$
\begin{aligned}
& \gamma_{\tau+1}^{A}-\gamma_{\tau}^{A}>0 \Leftrightarrow \frac{1}{1+\alpha \beta \varphi_{\tau+1}^{A}}-\frac{1}{1+\alpha \beta \varphi_{\tau}^{A}}>0 \quad \Leftrightarrow \quad \varphi_{\tau}^{A}>\varphi_{\tau+1}^{A} \\
& \Leftrightarrow q k_{\tau}^{A}+(1-q) k_{\tau+1}^{A}>q k_{\tau+1}^{A}+(1-q) k_{\tau+2}^{A}
\end{aligned}
$$

The last line is true because $k_{\tau}^{A}>k_{\tau+1}^{A}$, as we have discussed in relation to equation (7) on page 8. In spite of this indeterminacy, it is possible to show that the total extraction rate in the first phase, which we denote $d_{\tau}^{I}$ for clarity, increases with $\tau$ :

$$
\begin{aligned}
& d_{\tau+1}^{I}>d_{\tau}^{I} \Leftrightarrow s_{\tau+1} \gamma_{\tau+1}^{A}+1-s_{\tau+1}>s_{\tau} \gamma_{\tau}^{A}+1-s_{\tau} \\
& \Leftrightarrow \quad s_{\tau+1}\left(\gamma_{\tau+1}^{A}-1\right)>s_{\tau}\left(\gamma_{\tau}^{A}-1\right) \quad \Leftrightarrow \quad \frac{s_{\tau+1}}{s_{\tau}}<\frac{\gamma_{\tau}^{A}-1}{\gamma_{\tau+1}^{A}-1}
\end{aligned}
$$

The change in sign in the last line occurs because $\gamma_{\tau}^{A}<1$ for all $\tau$. The left-hand-side of the last inequality is smaller than one as $s_{\tau+1}<s_{\tau}$ by construction. The right-hand side, however, is larger than one: $\frac{\gamma_{\tau}^{A}-1}{\gamma_{\tau+1}^{A}-1}>1 \Leftrightarrow \gamma_{\tau}^{A}-1<\gamma_{\tau+1}^{A}-1 \Leftrightarrow \gamma_{\tau+1}^{A}>\gamma_{\tau}^{A}$. The first phase ends when $1-s_{\tau} \geq \frac{\gamma^{B}\left(1-\gamma_{\tau}^{A}\right)}{1-\gamma_{\tau}^{A} \gamma^{B}} \Leftrightarrow s_{\tau} \leq \frac{\alpha \beta}{1-(1-\alpha \beta) \gamma_{\tau}^{A}}$.

Consider the second phase. Equate the two non-binding best-replies $a_{\tau}=\gamma_{\tau}^{A}\left(1-b_{\tau}\right)$ and $b_{\tau}=\gamma^{B}\left(1-a_{\tau}\right)$ yields:

$$
a_{\tau}=\left(1-\gamma^{B}\left(1-a_{\tau}\right)\right) \gamma_{\tau}^{A} \quad \Leftrightarrow \quad a_{\tau}=\gamma_{\tau}^{A}-\gamma_{\tau}^{A} \gamma^{B}+a_{\tau} \gamma_{\tau}^{A} \gamma^{B} \quad \Leftrightarrow \quad a_{\tau}=\frac{\gamma_{\tau}^{A}\left(1-\gamma^{B}\right)}{1-\gamma_{\tau}^{A} \gamma^{B}}
$$

The case for player B is symmetrical. The total extraction rate is $d_{\tau}^{I I}=a_{\tau}+b_{\tau}=\frac{\gamma_{\tau}^{A}\left(1-\gamma^{B}\right)+\gamma^{B}\left(1-\gamma_{\tau}^{A}\right)}{1-\gamma_{\tau}^{A} \gamma^{B}}$. It is increasing with $\tau$ when the following holds:

$$
d_{\tau+1}^{I I}-d_{\tau}^{I I}=\frac{\left(\gamma_{\tau+1}^{A}-2 \gamma_{\tau+1}^{A} \gamma^{B}+\gamma^{B}\right)\left(1-\gamma_{\tau}^{A} \gamma^{B}\right)-\left(\gamma_{\tau}^{A}-2 \gamma_{\tau}^{A} \gamma^{B}+\gamma^{B}\right)\left(1-\gamma_{\tau+1}^{A} \gamma^{B}\right)}{\left(1-\gamma_{\tau+1}^{A} \gamma^{B}\right)\left(1-\gamma_{\tau}^{A} \gamma^{B}\right)}>0
$$

Because the denominator is positive $\left(\gamma_{\tau}^{A}<1\right.$ and $\left.\gamma^{B}<1\right)$ this is equivalent to:

$$
\left(\gamma_{\tau+1}^{A}-\gamma_{\tau}^{A}\right)\left(1+\left(\gamma^{B}\right)^{2}-2 \gamma^{B}\right)>0
$$

This inequality holds because the first bracket is positive, and since $\gamma^{B}=1-\alpha \beta$, the second 
bracket reduces to $(\alpha \beta)^{2}$, which is also positive.

Total extraction in the third phase is $d_{\tau}^{I I I}=s_{\tau}+\left(1-s_{\tau}\right) \gamma^{B}=1-\alpha \beta+\alpha \beta s_{\tau}$. This is declining as $\tau \rightarrow T$ simply due to the fact that $s_{\tau}>s_{\tau+1}$.

\section{A.2 Proof of Proposition 2}

In the following it will be useful to define the following auxiliary parameters:

$$
\varphi_{\tau}^{A} \equiv q k_{\tau}^{A}+(1-q) k_{\tau+1}^{A} \quad \text { and } \quad \varphi^{B} \equiv \frac{1}{1-\alpha \beta}
$$

We start by conjecturing that $V_{\tau}^{\text {coop }, i}$ and $V_{\tau}^{n c, i}$ are of the usual log-linear form and that the coefficients $k_{\tau}^{A}$ and $k_{\tau}^{B}$ take the form as described by equation (7). Taking the perspective of player A, her relevant value functions are:

$$
\begin{aligned}
V_{\tau}^{\text {coop }, A}\left(\lambda, x_{t}\right)=\ln \lambda(1-\alpha \beta)+\ln x_{t}+\ldots \\
\quad \ldots \beta\left(q\left[k_{\tau}^{A} \ln x_{t+1}+K_{\tau}^{c o o p, A}\right]+(1-q)\left[k_{\tau+1}^{A} \ln x_{t+1}+K_{\tau+1}^{A}\right]\right) \\
V_{\tau}^{n c, A}\left(\lambda, x_{t}\right)=\ln a_{\tau}+\ln x_{t}+\ldots \\
\ldots \beta\left(q\left[k_{\tau}^{A} \ln x_{t+1}+K_{\tau}^{n c, A}\right]+(1-q)\left[k_{\tau+1}^{A} \ln x_{t+1}+K_{\tau+1}^{A}\right]\right)
\end{aligned}
$$

Note that $x_{t+1}=M\left(\alpha \beta x_{t}\right)^{\alpha}$ under cooperation but $x_{t+1}=M\left(\left(1-a_{\tau}-b_{\tau}\right) x_{t}\right)^{\alpha}$ under noncooperation. Equating coefficients therefore shows that indeed $k_{\tau}^{A}=1+\alpha \beta \varphi_{\tau}^{A}$ as in the noncooperative case:

$$
\begin{aligned}
k_{\tau}^{A} \ln x_{t}+K_{\tau}^{c o o p}, A=\ln \lambda(1-\alpha \beta)+\ln x_{t}+\ldots \\
\ldots \beta\left(q\left[k_{\tau}^{A}\left(\ln M+\alpha \ln \alpha \beta+\alpha \ln x_{t}\right)+K_{\tau}^{c o o p, A}\right]+\ldots\right. \\
\left.\ldots(1-q)\left[k_{\tau+1}^{A}\left(\ln M+\alpha \ln \alpha \beta+\alpha \ln x_{t}\right)+K_{\tau+1}^{A}\right]\right)
\end{aligned}
$$

We have also:

$$
(1-\beta q) K_{\tau}^{c o o p, A}=\ln \lambda(1-\alpha \beta)+\beta\left((1-q) K_{\tau+1}^{A}+\varphi_{\tau}^{A}(\ln M+\alpha \ln \alpha \beta)\right)
$$

For the non-cooperative case, equating coefficients yields:

$$
(1-\beta q) K_{\tau}^{n c, A}=\ln a_{\tau}+\beta\left((1-q) K_{\tau+1}^{A}+\varphi_{\tau}^{A}\left(\ln M+\alpha \ln \left(1-a_{\tau}-b_{\tau}\right)\right)\right.
$$

Player A's participation constraint is satisfied when:

$$
V_{\tau}^{c o o p, A}\left(\lambda, x_{t}\right)-V_{\tau}^{n c, A}\left(x_{t}\right)=k_{\tau}^{A} \ln x_{t}+K_{\tau}^{c o o p, A}-\left(k_{\tau}^{A} \ln x_{t}+K_{\tau}^{n c, A}\right) \geq 0
$$

Clearly, the term $k_{\tau}^{A} \ln x_{t}$ cancels. Similarly, inspecting (A-1) and A-2 , we see that the term $K_{\tau+1}^{A}$, capturing the further development of the game, enters in the same manner in those two equations. Thus, it cancels as well. Therefore, we can define player A's gain from cooperation by $g^{A}:$ 


$$
g^{A}(\lambda)=\frac{1}{1-\beta q}\left(\ln \lambda+\ln \left(\frac{1-\alpha \beta}{a_{\tau}}\right)+\alpha \beta \varphi_{\tau}^{A} \ln \left(\frac{\alpha \beta}{1-a_{\tau}-b_{\tau}}\right)\right)
$$

Whether this $g^{A}(\lambda)>0$ is in fact independent of the current stock level and the future development of the game.

\section{There is not always scope for cooperation}

To see that there cannot always be scope for cooperation, note that $g^{A}(\lambda)$ is increasing in $\lambda$ and reaches its maximum at $\lambda=1$. We now show that $g^{A}(1)<0$ as $\alpha \beta \rightarrow 1$ or $q \rightarrow 0$, so that player A would not join an agreement, even if she were offered the entire harvest.

To show that $g^{A}(1)<0$ as $q \rightarrow 0$, consider A's gain from cooperation at stage $T-1$. At this stage we have $\lim _{q \rightarrow 0} \varphi_{T-1}^{A}=0$ and consequently $a_{T-1}=s_{T-1}$ (Player A will harvest all she can before she loses the resource for sure). We have $g^{A}(1)<0 \Leftrightarrow \ln \left(\frac{1-\alpha \beta}{a_{\tau}}\right)<0 \Leftrightarrow 1-\alpha \beta<a_{T-1}$. When $s_{T-1}<1-\alpha \beta$ we need to consider the game at stage $\tau<T-1$. But from backward induction, it follows that $a_{\tau}=s_{\tau}$ for all $\tau$ and consequently $b_{\tau}=\left(1-s_{\tau}\right)(1-\alpha \beta)$. Now there will be some $\tau$ at which $s_{\tau}>1-\alpha \beta$ and since $\varphi_{\tau}^{A}$ is bounded above by $\frac{1}{1-\alpha \beta}$ we know that at some stage $\left(\right.$ as $\left.s_{\tau} \rightarrow 1\right)$ we have $\ln (1-\alpha \beta)-\left(1+\alpha \beta \varphi_{\tau}^{A}\right) \ln s_{\tau}<0$ and thus $g^{A}(1)<0$.

Consider now the case when $\alpha \beta \rightarrow 1$. We have that $g^{A}(1)<0$ whenever:

$$
\lim _{\alpha \beta \rightarrow 1} \ln \left(\frac{1-\alpha \beta}{a_{\tau}}\right)+\alpha \beta \varphi_{\tau}^{A} \ln \left(\frac{\alpha \beta}{1-a_{\tau}-b_{\tau}}\right)<0
$$

To evaluate this limit, we need a few building stones. Consider first $k_{\tau}^{A}, \varphi_{\tau}^{A}$ and $\gamma_{\tau}^{A}$, where we use L'Hôpital's rule to show that these terms converge to some constant $\kappa$ :

$$
\begin{aligned}
\lim _{\alpha \beta \rightarrow 1} k_{\tau}^{A} & =\lim _{\alpha \beta \rightarrow 1}\left[\frac{1}{1-\alpha \beta}\left(1-\left(\frac{\alpha \beta(1-q)}{1-\alpha \beta q}\right)^{T-\tau}\right)\right] \\
& =\frac{\lim _{\alpha \beta \rightarrow 1}\left[-(T-\tau)\left(\frac{\alpha \beta(1-q)}{1-\alpha \beta q}\right)^{T-\tau-1}\left(\frac{1-q}{(1-\alpha \beta q)^{2}}\right)\right]}{\lim _{\alpha \beta \rightarrow 1}-1} \\
& =(T-\tau) \lim _{\alpha \beta \rightarrow 1}\left[\left(\frac{\alpha \beta(1-q)}{1-\alpha \beta q}\right)^{T-\tau-1}\right] \lim _{\alpha \beta \rightarrow 1}\left[\frac{1-q}{(1-\alpha \beta q)^{2}}\right]=\frac{T-\tau}{1-q}=\kappa_{1}>0 \\
\lim _{\alpha \beta \rightarrow 1} \varphi_{\tau}^{A} & =\lim _{\alpha \beta \rightarrow 1} q k_{\tau}^{A}+(1-q) k_{\tau+1}^{A}=q \frac{(T-\tau)}{(1-q)}+T-\tau-1=\kappa_{2}>0 \\
\lim _{\alpha \beta \rightarrow 1} \gamma_{\tau}^{A} & =\lim _{\alpha \beta \rightarrow 1} \frac{1}{1+\alpha \beta \varphi^{A}}=\kappa_{3}>0 \\
\lim _{\alpha \beta \rightarrow 1} \gamma^{B} & =\lim _{\alpha \beta \rightarrow 1}(1-\alpha \beta)=0
\end{aligned}
$$

And therefore:

$$
\lim _{\alpha \beta \rightarrow 1} a_{\tau}=\left\{\begin{array}{lll}
\lim _{\alpha \beta \rightarrow 1} s_{\tau} \gamma_{\tau}^{A} & =s_{\tau} \kappa_{3} & \text { Phase I } \\
\lim _{\alpha \beta \rightarrow 1} \frac{\gamma_{\tau}^{A}\left(1-\gamma^{B}\right)}{1-\gamma_{\tau}^{A} \gamma^{B}} & =\kappa_{3} & \text { Phase II } \\
\lim _{\alpha \beta \rightarrow 1} s_{\tau} & =s_{\tau}
\end{array} \quad \text { Phase III } \quad \lim _{\alpha \beta \rightarrow 1} b_{\tau}= \begin{cases}\lim _{\alpha \beta \rightarrow 1} 1-s_{\tau} & =1-s_{\tau} \\
\lim _{\alpha \beta \rightarrow 1} \frac{\gamma^{B}\left(1-\gamma_{\tau}^{A}\right)}{1-\gamma_{\tau}^{A} \gamma^{B}} & =0 \\
\lim _{\alpha \beta \rightarrow 1}\left(1-s_{\tau}\right) \gamma^{B} & =0\end{cases}\right.
$$


Hence, $\lim _{\alpha \beta \rightarrow 1} \ln \left(\frac{1-\alpha \beta}{a_{\tau}}\right)=-\infty$, but $\lim _{\alpha \beta \rightarrow 1} \alpha \beta \varphi_{\tau}^{A} \ln \left(\frac{\alpha \beta}{1-a_{\tau}-b_{\tau}}\right)=\kappa_{2} \ln \left(\frac{1}{1-s_{\tau} \kappa_{3}}\right)$ in Phase I, $=\kappa_{2} \ln \left(\frac{1}{1-\kappa_{3}}\right)$ in Phase II, and $=\kappa_{2} \ln \left(\frac{1}{1-s_{\tau}}\right)$ in Phase III. In any case, it is bounded above so that the entire term $\left[\ln \left(\frac{1-\alpha \beta}{a_{\tau}}\right)+\alpha \beta \varphi_{\tau}^{A} \ln \left(\frac{\alpha \beta}{1-a_{\tau}-b_{\tau}}\right)\right]$ must be smaller than zero as claimed.

\section{Derivation of $\lambda_{\min }^{A}$ and $\lambda_{\max }^{B}$}

Now consider the situation where $\alpha \beta$ take values such that $g^{A}\left(\lambda_{m i n}^{A}\right)=0$ exists. By re-writing equation $\mathrm{A}-3$ and taking exponents on both sides, we get:

$$
\lambda_{\min }^{A}=\frac{a_{\tau}}{1-\alpha \beta}\left(\frac{1-a_{\tau}-b_{\tau}}{\alpha \beta}\right)^{\alpha \beta \varphi_{\tau}^{A}}
$$

By parallel reasoning, player B's participation constraint is satisfied when $V_{\tau}^{\text {coop, } B}-V_{\tau}^{n c, B} \geq 0$ and $\lambda_{\max }^{B}$, the maximum share that player B would be willing to give to player $\mathrm{A}$ and still harvest cooperatively is - if it exists - defined by:

$$
\lambda_{\max }^{B}=1-\frac{b_{\tau}}{1-\alpha \beta}\left(\frac{1-a_{\tau}-b_{\tau}}{\alpha \beta}\right)^{\alpha \beta \varphi^{B}}
$$

\section{Cooperation possibilities are not constrained by the available harvest shares}

At any stage $\tau$, player $\mathrm{A}$ needs to harvest at least $(1-\alpha \beta) \lambda_{\min }^{A}$ to join the agreement. This is less than he would harvest under non-cooperation:

$$
(1-\alpha \beta) \lambda_{\min }^{A}<a_{\tau} \Leftrightarrow a_{\tau}\left(\frac{1-a_{\tau}-b_{\tau}}{\alpha \beta}\right)^{\alpha \beta \varphi_{\tau}^{A}}<a_{\tau} \Leftrightarrow\left(\frac{1-a_{\tau}-b_{\tau}}{\alpha \beta}\right)^{\alpha \beta \varphi_{\tau}^{A}}<1
$$

The last statement is true because $1-a_{\tau}-b_{\tau}=1-d^{n c}<1-d^{*}=\alpha \beta$ and $\alpha \beta \varphi>0$ and a function $x^{y}<1$ when $x<1$ and $y>0$. For player $\mathrm{B}$, the argumentation is parallel:

$$
(1-\alpha \beta)\left(1-\lambda_{\max }^{B}\right)<b_{\tau} \quad \Leftrightarrow \quad b_{\tau}\left(\frac{1-a_{\tau}-b_{\tau}}{\alpha \beta}\right)^{\alpha \beta \varphi^{B}}<b_{\tau} \Leftrightarrow\left(\frac{1-a_{\tau}-b_{\tau}}{\alpha \beta}\right)^{\alpha \beta \varphi^{B}}<1
$$

As $a_{\tau} \leq s_{\tau}$ and $b_{\tau} \leq 1-s_{\tau}$ by construction, it follows immediately that cooperation possibilities are not constrained by the accessible harvest shares.

This completes the proof of Proposition 2 .

\section{A.3 Proof of Proposition 3}

To show how the extraction pattern changes with we need to derive $\frac{\partial \gamma_{\tau}^{A}}{\partial q}\left(k^{B}\right.$ and thus $\gamma^{B}$ do not depend on $q$ ). In this derivation, we will again employ the following auxiliary parameters to make the derivations more concise.

$$
\varphi_{\tau}^{A} \equiv q k_{\tau}^{A}+(1-q) k_{\tau+1}^{A} \quad \text { and } \quad \varphi^{B} \equiv \frac{1}{1-\alpha \beta}
$$

To start, consider $\frac{\partial k_{\tau}^{A}}{\partial q}$ : 


$$
\begin{aligned}
\frac{\partial k_{\tau}^{A}}{\partial q} & =\frac{-(T-\tau)\left(\frac{\alpha \beta(1-q)}{1-\alpha \beta q}\right)^{T-\tau-1}\left(\frac{-\alpha \beta(1-\alpha \beta q)-\alpha \beta(1-q)(-\alpha \beta)}{(1-\alpha \beta q)^{2}}\right)}{1-\alpha \beta} \\
& =-(T-\tau)\left(\frac{\alpha \beta(1-q)}{1-\alpha \beta q}\right)^{T-\tau-1}\left(\frac{-\alpha \beta}{(1-\alpha \beta q)^{2}}\right)>0
\end{aligned}
$$

The above follows from $T>\tau$ and because the term $\left(\frac{\alpha \beta(1-q)}{1-\alpha \beta q}\right)>0$ as $\alpha, \beta$, and $q \in(0,1)$. As a consequence:

$$
\begin{aligned}
\frac{\partial \varphi_{\tau}^{A}}{\partial q} & =k_{\tau}^{A}+q \frac{\partial k_{\tau}^{A}}{\partial q}-k_{\tau+1}^{A}+(1-q) \frac{\partial k_{\tau+1}^{A}}{\partial q} \\
& =k_{\tau}^{A}-k_{\tau+1}^{A}+q\left(\frac{\partial k_{\tau}^{A}}{\partial q}-\frac{\partial k_{\tau+1}^{A}}{\partial q}\right)+\frac{\partial k_{\tau+1}^{A}}{\partial q}>0
\end{aligned}
$$

Here, we have to show that $\frac{\partial k_{\tau}^{A}}{\partial q}>\frac{\partial k_{\tau+1}^{A}}{\partial q}$ for all $k_{\tau}^{A}>k_{\tau+1}^{A}$ and $q>0$ and $\frac{\partial k_{\tau+1}^{A}}{\partial q}>0$. From A-4 we have:

$$
\begin{aligned}
\frac{\partial k_{\tau}^{A}}{\partial q}-\frac{\partial k_{\tau+1}^{A}}{\partial q}>0 & \Leftrightarrow(T-\tau-1)\left(\frac{\alpha \beta(1-q)}{1-\alpha \beta q}\right)^{T-\tau-2}-(T-\tau)\left(\frac{\alpha \beta(1-q)}{1-\alpha \beta q}\right)^{T-\tau-1}>0 \\
& \Leftrightarrow(T-\tau-1)\left(\frac{1-\alpha \beta q}{\alpha \beta(1-q)}\right)>T-\tau \\
& \Leftrightarrow T-\tau-1>\alpha \beta(T-\tau-q)
\end{aligned}
$$

This is true because $\alpha, \beta$, and $q \in(0,1)$. This implies:

$$
\frac{\partial \gamma_{\tau}^{A}}{\partial q}=\frac{-\alpha \beta \frac{\partial \varphi_{\tau}^{A}}{\partial q}}{\left(1+\alpha \beta \varphi^{A}\right)^{2}}<0
$$

Furthermore, we need to derive $\frac{\partial \gamma_{T}^{A}}{\partial T}\left(k^{B}\right.$ and thus $\varphi^{B}$ and $\gamma^{B}$ do not depend on T.). Again, consider first $\frac{\partial k_{\tau}^{A}}{\partial T}$ :

$$
\frac{\partial k_{\tau}^{A}}{\partial T}=\frac{-1}{1-\alpha \beta}\left(\frac{\alpha \beta(1-q)}{1-\alpha \beta q}\right)^{T-\tau} \ln \left(\frac{\alpha \beta(1-q)}{1-\alpha \beta q}\right)>0
$$

Since $\left(\frac{\alpha \beta(1-q)}{1-\alpha \beta q}\right)<1$, the logarithm yields a negative number, so that the entire term is positive. Consequently:

$$
\begin{aligned}
\frac{\partial \varphi_{\tau}^{A}}{\partial T} & =q \frac{\partial k_{\tau}^{A}}{\partial T}+(1-q) \frac{\partial k_{\tau+1}^{A}}{\partial T}>0 \\
\frac{\partial \gamma_{\tau}^{A}}{\partial T} & =\frac{-\alpha \beta \frac{\partial \varphi_{\tau}^{A}}{\partial T}}{\left(1+\alpha \beta \varphi^{A}\right)^{2}}<0
\end{aligned}
$$

In the first phase, player B is harvesting the entire share available to him $\left(b_{\tau}^{I}=1-s_{\tau}\right)$. Hence his extraction rate does not depend on $q$. It does depend on $T$, however, since $s_{\tau}=\frac{T-\tau}{T}$. $s_{\tau}$ 
increases with $T$ so that $b_{\tau}^{I}$ decreases. From (A-6), it follows that player A's and thus the total extraction rate is decreasing in $q$. Regarding an increase in $T$, we see from $\mathrm{A}-9$ that $\gamma_{\tau}^{A}$ decreases, but $s_{\tau}$ increases. The total effect is still negative:

$$
\begin{aligned}
\frac{\partial d_{\tau}^{I}}{\partial T} & =\frac{\partial s_{\tau}}{\partial T} \gamma_{\tau}^{A}+s_{\tau} \frac{\partial \gamma_{\tau}^{A}}{\partial T}-\frac{\partial s_{\tau}}{\partial T}<0 \\
& \Leftrightarrow \frac{\partial s_{\tau}}{\partial T} \gamma_{\tau}^{A}+s_{\tau} \frac{\partial \gamma_{\tau}^{A}}{\partial T}-\frac{\partial s_{\tau}}{\partial T}<0 \\
& \Leftrightarrow s_{\tau} \frac{\partial \gamma_{\tau}^{A}}{\partial T}<\left(1-\gamma_{\tau}^{A}\right) \frac{\partial s_{\tau}}{\partial T}
\end{aligned}
$$

This is true because $\frac{\partial \gamma_{\tau}^{A}}{\partial T}<0$ and all other terms are positive (and $\gamma_{\tau}^{A}<1$ ).

In the second phase, we have $d^{I I}=\frac{\gamma_{\tau}^{A}+\gamma^{B}-2 \gamma_{\tau}^{A} \gamma^{B}}{1-\gamma_{\tau}^{A} \gamma^{B}}$. Consequently:

$$
\begin{aligned}
\frac{\partial d_{\tau}^{I I}}{\partial q} & =\frac{\left(\frac{\partial \gamma_{\tau}^{A}}{\partial q}-\frac{\partial 2 \gamma_{\tau}^{A} \gamma^{B}}{\partial q}\right)\left(1-\gamma_{\tau}^{A} \gamma^{B}\right)-\frac{\partial\left(1-\gamma_{\tau}^{A} \gamma^{B}\right)}{\partial q}\left(\gamma_{\tau}^{A}+\gamma^{B}-2 \gamma_{\tau}^{A} \gamma^{B}\right)}{\left(1-\gamma_{\tau}^{A} \gamma^{B}\right)^{2}} \\
& =\frac{\left(\frac{\partial \gamma_{\tau}^{A}}{\partial q}-2 \gamma^{B} \frac{\partial \gamma_{\tau}^{A}}{\partial q}\right)\left(1-\gamma_{\tau}^{A} \gamma^{B}\right)+\gamma^{B} \frac{\partial \gamma_{\tau}^{A}}{\partial q}\left(\gamma_{\tau}^{A}+\gamma^{B}-2 \gamma_{\tau}^{A} \gamma^{B}\right)}{\left(1-\gamma_{\tau}^{A} \gamma^{B}\right)^{2}} \\
& =\frac{\left(1-\gamma^{B}\right)^{2} \frac{\partial \gamma_{\tau}^{A}}{\partial q}}{\left(1-\gamma_{\tau}^{A} \gamma^{B}\right)^{2}}=\frac{(\alpha \beta)^{2} \frac{\partial \gamma_{\tau}^{A}}{\partial q}}{\left(1-\gamma_{\tau}^{A} \gamma^{B}\right)^{2}}
\end{aligned}
$$

As we know, $(\alpha \beta)^{2}>0$ and $\frac{\partial \gamma_{\tau}^{A}}{\partial q}<0$ (confer $\left.\mathrm{A}-6\right)$, which implies a negative nominator and a positive denominator. Consequently, the total extraction is decreasing in $q$.

Similarly:

$$
\begin{aligned}
\frac{\partial d_{\tau}^{I I}}{\partial T} & =\frac{\left(\frac{\partial \gamma_{\tau}^{A}}{\partial T}-\frac{\partial 2 \gamma_{\tau}^{A} \gamma^{B}}{\partial T}\right)\left(1-\gamma_{\tau}^{A} \gamma^{B}\right)-\frac{\partial\left(1-\gamma_{\tau}^{A} \gamma^{B}\right)}{\partial T}\left(\gamma_{\tau}^{A}+\gamma^{B}-2 \gamma_{\tau}^{A} \gamma^{B}\right)}{\left(1-\gamma_{\tau}^{A} \gamma^{B}\right)^{2}} \\
& =\frac{(\alpha \beta)^{2} \frac{\partial \gamma_{\tau}^{A}}{\partial T}}{\left(1-\gamma_{\tau}^{A} \gamma^{B}\right)^{2}}
\end{aligned}
$$

Again, $(\alpha \beta)^{2}>0$ and $\frac{\partial \gamma_{\tau}^{A}}{\partial T}<0$ (following from $(\mathrm{A}-9)$ ), which implies a negative nominator and a positive denominator. Consequently, the total extraction is decreasing in $T$ in the second phase.

In the third phase, neither player's extraction rate reacts to changes in $q$. Instead, both player's extration rates depend on $T$ as $a_{\tau}^{I I I}=s_{\tau}$ and $b_{\tau}^{I I I}=\left(1-s_{\tau}\right) \gamma^{B}$. As an increase in $T$ means a larger $s_{\tau}$, the extraction rate of player A increases in $T$, and consequently player B's extraction rate decreases. The total effect is increasing, as player B's declining effect is smaller than the effect of player $\mathrm{A}(1-\alpha \beta<1)$.

\section{A.4 Proof of Proposition 4}

To see that an increase in $\alpha$ or $\beta$ (and hence $\alpha \beta$ ) leads to a lower total extraction rate in all phases, we first need to establish $\frac{\partial \gamma_{\tau}^{A}}{\partial \alpha \beta}<0$ and $\frac{\partial \gamma^{B}}{\partial \alpha \beta}<0$. As $\gamma^{B}=1-\alpha \beta$, the sign of the latter derivative 
is obvious. For $\gamma_{\tau}^{A}=\left(1+\alpha \beta\left(q k_{\tau}^{A}+(1-q) k_{\tau+1}^{A}\right)\right)^{-1}$, it is more involved. We first need to show that $\frac{\partial k_{\tau}^{A}}{\partial \alpha \beta}>0$ :

$$
\begin{aligned}
& \frac{\partial k_{\tau}^{A}}{\partial \alpha \beta}=\frac{\left(1-\left(\frac{\alpha \beta(1-q)}{1-\alpha \beta q}\right)^{T-\tau}\right)-(T-\tau)\left(\frac{\alpha \beta(1-q)}{1-\alpha \beta q}\right)^{T-\tau-1}\left(\frac{(1-q)(1-\alpha \beta q)+\alpha \beta q(1-q)}{(1-\alpha \beta q)^{2}}\right)(1-\alpha \beta)}{(1-\alpha \beta)^{2}}>0 \\
& \Leftrightarrow \quad\left(1-\left(\frac{\alpha \beta(1-q)}{1-\alpha \beta q}\right)^{T-\tau}\right)>(T-\tau)\left(\frac{\alpha \beta(1-q)}{1-\alpha \beta q}\right)^{T-\tau-1}\left(\frac{(1-q)(1-\alpha \beta)}{(1-\alpha \beta q)^{2}}\right) \\
& \Leftrightarrow \quad\left(\frac{\alpha \beta(1-q)}{1-\alpha \beta q}\right)^{\tau-T}-1>(T-\tau)\left(\frac{1-\alpha \beta q}{\alpha \beta(1-q)}\right) \frac{(1-q)(1-\alpha \beta)}{(1-\alpha \beta q)^{2}} \\
& \Leftrightarrow \quad\left(\frac{1-\alpha \beta q}{\alpha \beta-\alpha \beta q}\right)^{T-\tau}-1>(T-\tau) \frac{1-\alpha \beta}{\alpha \beta(1-\alpha \beta q)}
\end{aligned}
$$

We know that this holds because at $\tau=T-1$ (the largest value that $\tau$ can take at which $k_{\tau}^{A}$ is still strictly positive), the last line reads: $\frac{1-\alpha \beta q}{\alpha \beta-\alpha \beta q}-1>\frac{1-\alpha \beta}{\alpha \beta(1-\alpha \beta q)}$. This can be transformed to $q(1-\alpha \beta)^{2}>0$ which is true because $q<1$ and $\alpha \beta<1$. For $\tau>T-1$ the left-hand-side (LHS) of the above inequality is growing exponentially while the right-hand-side (RHS) is growing linearly.

The subsequent comparative static results follow immediately from $\frac{\partial k_{\tau}^{A}}{\partial \alpha \beta}>0$ :

$$
\begin{aligned}
& \frac{\partial\left[q k_{\tau}^{A}+(1-q) k_{\tau+1}^{A}\right]}{\partial \alpha \beta}=q \frac{\partial k_{\tau}^{A}}{\partial \alpha \beta}+(1-q) \frac{\partial k_{\tau+1}^{A}}{\partial \alpha \beta}>0 \\
& \frac{\partial \gamma_{\tau}^{A}}{\partial \alpha \beta}=\frac{-\left[q k_{\tau}^{A}+(1-q) k_{\tau+1}^{A}\right]-\alpha \beta \frac{\partial\left[q k_{\tau}^{A}+(1-q) k_{\tau+1}^{A}\right]}{\partial \alpha \beta}}{\left(1+\alpha \beta\left[q k_{\tau}^{A}+(1-q) k_{\tau+1}^{A}\right]\right)^{2}}<0
\end{aligned}
$$

In the first phase, Player $B$ 's extraction rate is given by $b_{\tau}^{I}=1-s_{\tau}$ and does not depend on $\alpha \beta$. Player $A$ 's extraction rate in the first phase is given by $a_{\tau}^{I}=s_{\tau} \gamma_{\tau}^{A}$ and $\frac{\partial a_{\tau}^{I}}{\partial \alpha \beta}=s_{\tau} \frac{\partial \gamma_{\tau}^{A}}{\partial \alpha \beta}<0$, which follows from $\mathrm{A}-10$.

In Phase II, total extraction is $d_{\tau}^{I I}=a_{\tau}^{I I}+b_{\tau}^{I I}=\frac{\gamma_{\tau}^{A}\left(1-\gamma^{B}\right)}{1-\gamma_{\tau}^{A} \gamma^{B}}+\frac{\gamma^{B}\left(1-\gamma_{\tau}^{A}\right)}{1-\gamma_{\tau}^{A} \gamma^{B}}=\frac{\gamma_{\tau}^{A}+\gamma^{B}-2 \gamma_{\tau}^{A} \gamma^{B}}{1-\gamma_{\tau}^{A} \gamma^{B}}$. Consequently:

$$
\begin{aligned}
\frac{\partial d_{\tau}^{I I}}{\partial \alpha \beta} & =\frac{\left(\frac{\partial \gamma_{\tau}^{A}}{\partial \alpha \beta}+\frac{\partial \gamma^{B}}{\partial \alpha \beta}-\frac{\partial 2 \gamma_{\tau}^{A} \gamma^{B}}{\partial \alpha \beta}\right)\left(1-\gamma_{\tau}^{A} \gamma^{B}\right)-\frac{\partial\left(1-\gamma_{\tau}^{A} \gamma^{B}\right)}{\partial \alpha \beta}\left(\gamma_{\tau}^{A}+\gamma^{B}-2 \gamma_{\tau}^{A} \gamma^{B}\right)}{\left(1-\gamma_{\tau}^{A} \gamma^{B}\right)^{2}} \\
& =\frac{\left(\frac{\partial \gamma_{\tau}^{A}}{\partial \alpha \beta}-1+2 \gamma_{\tau}^{A}-2 \gamma^{B} \frac{\partial \gamma_{\tau}^{A}}{\partial \alpha \beta}\right)\left(1-\gamma_{\tau}^{A} \gamma^{B}\right)-\left(\gamma_{\tau}^{A}-\gamma^{B} \frac{\partial \gamma_{\tau}^{A}}{\partial \alpha \beta}\right)\left(\gamma_{\tau}^{A}+\gamma^{B}-2 \gamma_{\tau}^{A} \gamma^{B}\right)}{\left(1-\gamma_{\tau}^{A} \gamma^{B}\right)^{2}} \\
& =\frac{(\alpha \beta)^{2} \frac{\partial \gamma_{\tau}^{A}}{\partial \alpha \beta}-\left(1-\gamma_{\tau}^{A}\right)^{2}}{\left(1-\gamma_{\tau}^{A} \gamma^{B}\right)^{2}}
\end{aligned}
$$

As $(\alpha \beta)^{2}>0, \frac{\partial \gamma_{\tau}^{A}}{\partial \alpha \beta}<0$ and $\left(1-\gamma_{\tau}^{A}\right)^{2}>0$, the nominator is negative. Additionally, the denominator is $\left(1-\gamma_{\tau}^{A} \gamma^{B}\right)^{2}>0$, and consequently the total extraction is decreasing in $\alpha \beta$ in the second phase.

In the third phase, player $A$ 's extraction rate is independent of $\alpha$ and $\beta$, and thus changes in those do not affect her extraction rate. However, player $B$ 's extraction is linearly dependent on both $\alpha$ and $\beta$ as $b_{\tau}=\left(1-s_{\tau}\right) 1-\alpha \beta$, and an increase in those parameters results in a decrease in player $B$ 's, and consequently in the total extraction rate. 
[This section is intended as online appendix.]

\section{A.5 R-code to produce Figures and Tables in main text}

Here we provide the computer codes that can be used to replicate the Figures and the Tables in the main text and to explore the model for different combinations of parameter values. The codes are written in "R" and consist of two files, one which produces the Figures and Tables (named ShiftStockFigTab.R) and one which is used to generate the vectors that are plotted with help of the first code (named ShiftStockGenVec.R).

The file ShiftStockFigTab.R to produce the Figures and tables:

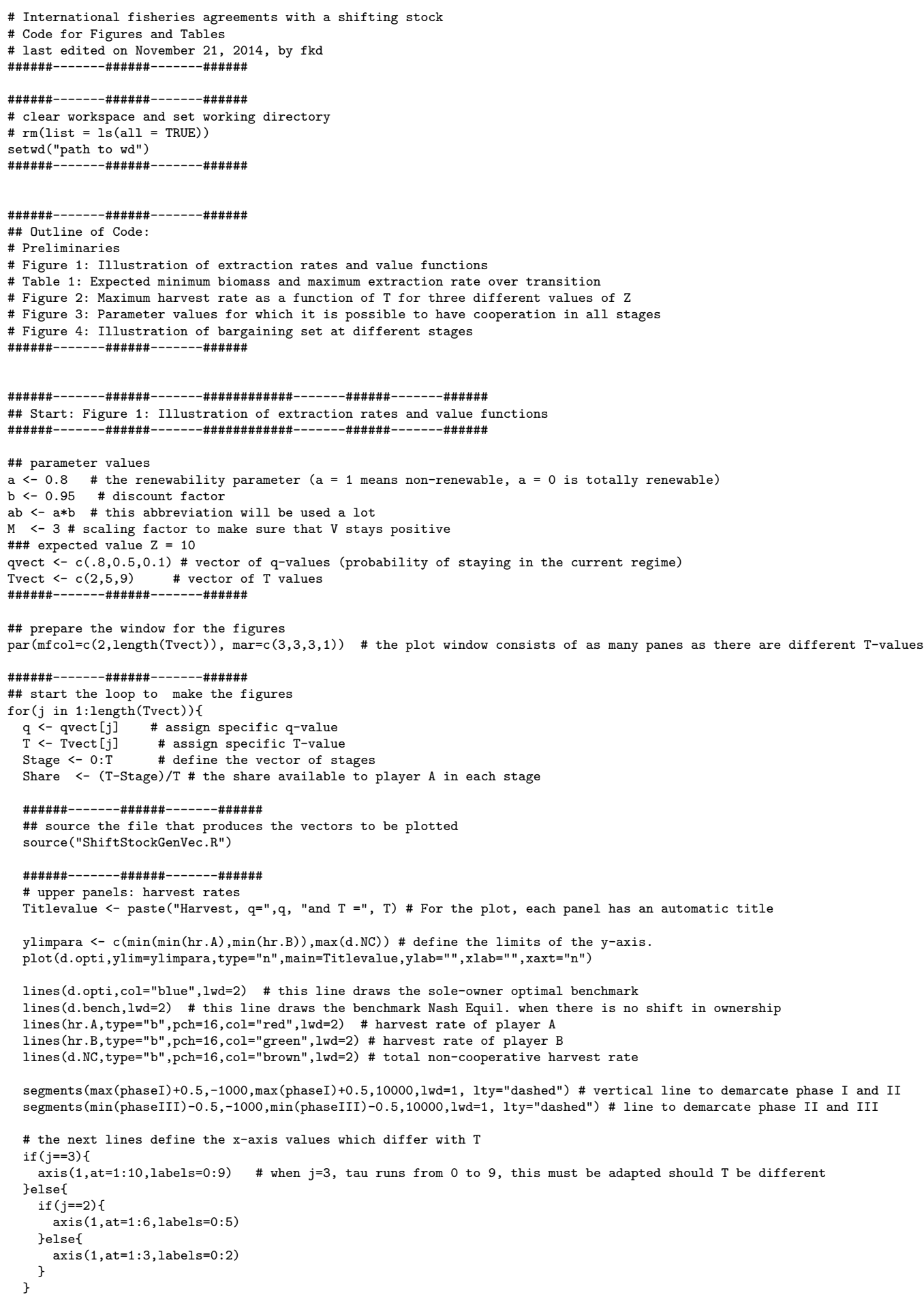


lg.text <- c("sole-owner benchm", "benchmark Nash", "player A NC", "player B NC", "total NC") \# text for legend legend ("bottomleft", lg.text, pch = 16, col=c ("blue", "black", "red", "green", "brown"), cex=0.7) \# place legend in plot

rm(lg.text,ylimpara) \# remove the variables lg.text and ylimpara

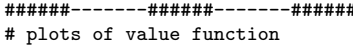

\#\# create the vectors for plotting

for $(j$ in $1: 3)\{\# j$-loop through the different $z$ values

$\mathrm{Z}<-\operatorname{Zvect}[j]$

Tvect $<-1:(Z-1) \#$ for a given $Z$, return the possible integer values of $T$

dmaxPlot <- rep(NA,length(Tvect)) \# create an empty vector to later store the values if max hatrvest rate dmax

for( $i$ in $1:$ length(Tvect)) $\{\# i$-loop through the different values of $T$ (total number of stages)

$\mathrm{T}<-$ Tvect $[\mathrm{i}]$

$q<-1-(T / Z) \#$ find the value of $q$ that corresponds to given $Z$ and $T$

Stage $<-0: T$

Share <- (T-Stage)/T

source("ShiftStockGenVec.R") \# source the file that creates the vector of total harvest rates

dmaxPlot [i] <- $\max (\mathrm{d} . \mathrm{NC})$ \# find the maximum harvest rate for a given combination of $\mathrm{q}, \mathrm{T}$, and

if $(j==1)\{$

dmaxPlot.10 <- dmaxPlot \# maximum harvest rate when $\mathrm{Z}=10$ 


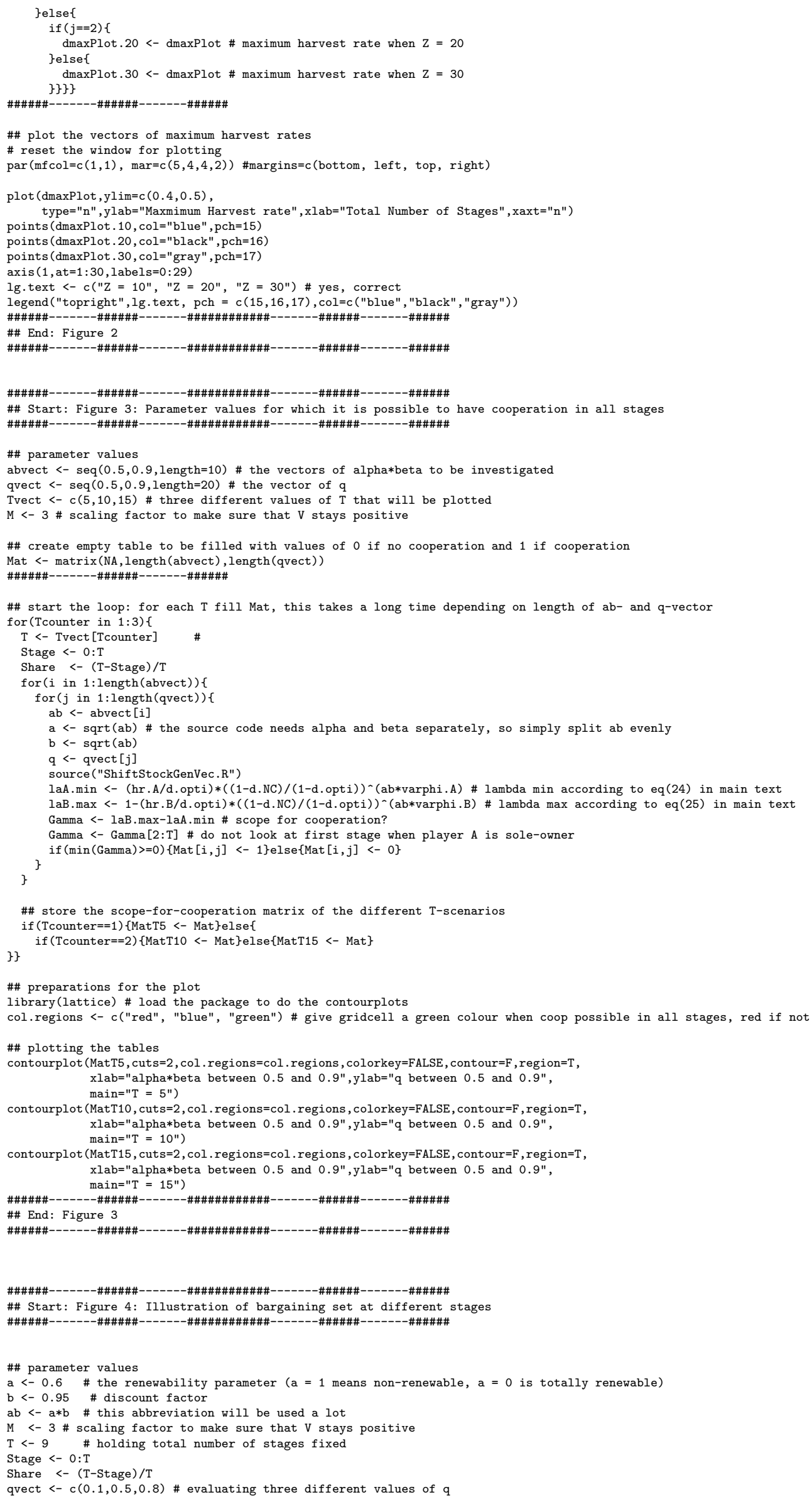

\#\# store the scope-for-cooperation matrix of the different T-scenarios if (Tcounter==1) \{MatT5 <- Mat\}else

if $($ Tcounter $=2)$ \{MatT10 <- Mat\}else $\{$ MatT15 <- Mat $\}$

\#\# preparations for the plot

library(lattice) \# load the package to do the contourplots

col.regions <- c("red", "blue", "green") \# give gridcell a green colour when coop possible in all stages, red if not

\#\# plotting the tables

contourplot (MatT5, cuts=2, col. regions=col. regions, , colorkey=FALSE, contour=F, region=T $\mathrm{xlab}=" \mathrm{alpha*beta}$ between 0.5 and $0.9 ", \mathrm{ylab}=" \mathrm{q}$ between 0.5 and $0.9 "$ ", main=" $\mathrm{T}=5 "$ )

contourplot (MatT10, cuts=2, col. regions=col. regions, colorkey=FALSE, contour=F, region= $\mathrm{T}$, $\mathrm{xlab}=" \mathrm{alpha} *$ beta between 0.5 and $0.9 "$ "ylab="q between 0.5 and $0.9 "$, main="T = 10")

contourplot (MatT15, cuts $=2$, col regions $=\mathrm{col}$. regions, colorkey=FALSE, contour=F, region=T, $\mathrm{xlab}=" \mathrm{alpha} *$ beta between 0.5 and $0.9 ", \mathrm{ylab}=" \mathrm{q}$ between 0.5 and $0.9 "$,

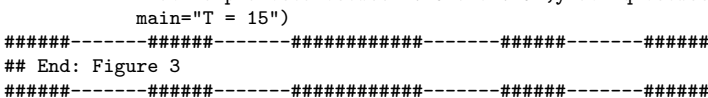

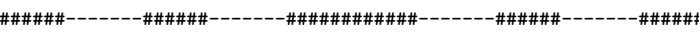

\#\# Start: Figure 4: Illustration of bargaining set at different stages

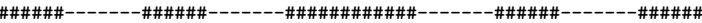

\#\# parameter values

a $<-0.6 \#$ the renewability parameter ( $\mathrm{a}=1$ means non-renewable, $\mathrm{a}=0$ is totally renewable)

b $<-0.95 \#$ discount factor

$\mathrm{ab}<-\mathrm{a} * \mathrm{~b} \#$ this abbreviation will be used a lot

$M<-3$ \# scaling factor to make sure that $V$ stays positive

T<- $9 \quad$ \# holding total number of stages fixed

Stage $<-0: T$

Share $<-($ T-Stage $) / T$

qvect $<-c(0.1,0.5,0.8)$ \# evaluating three different values of $q$ 


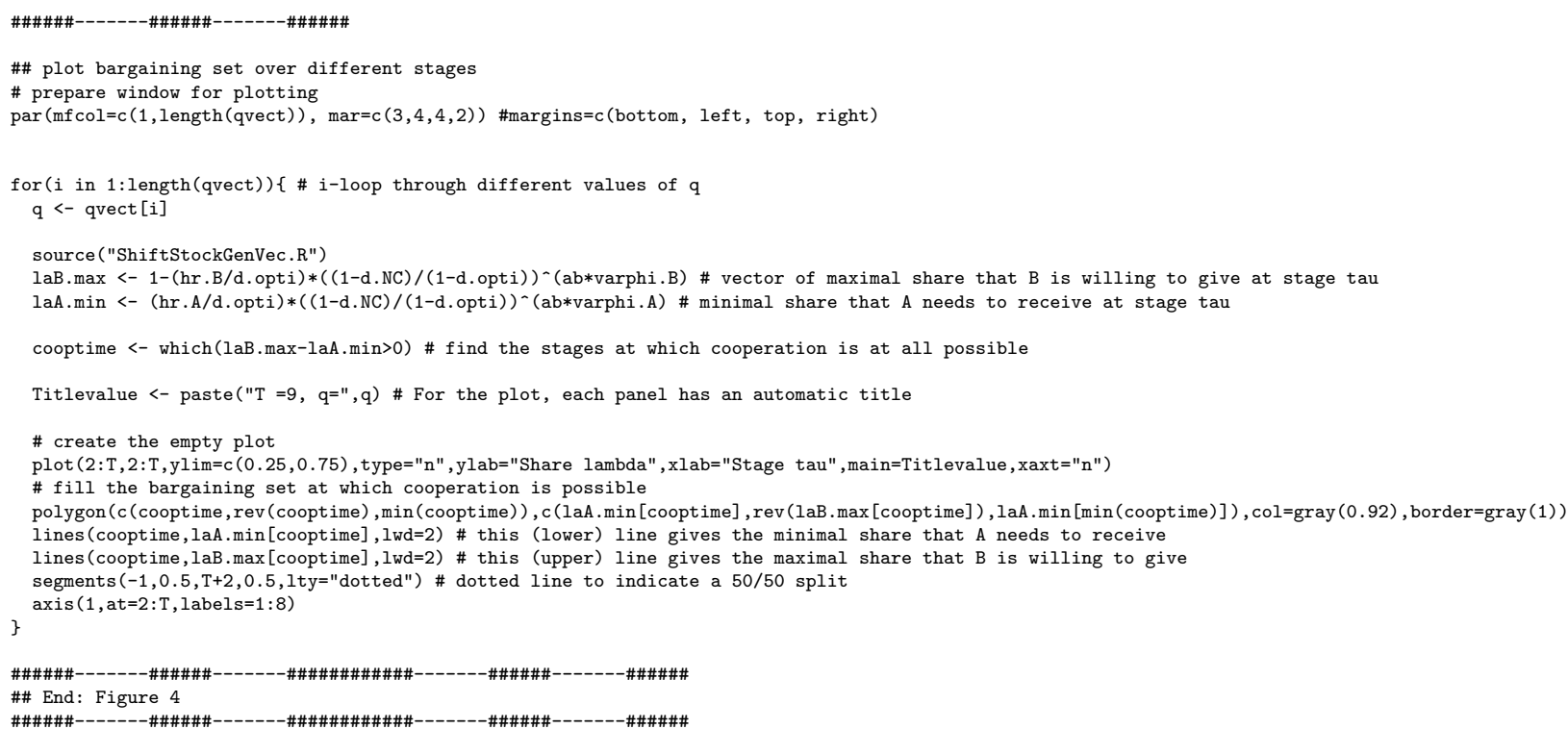

The file ShiftStockGenVec.R that generates the vectors to be plotted:

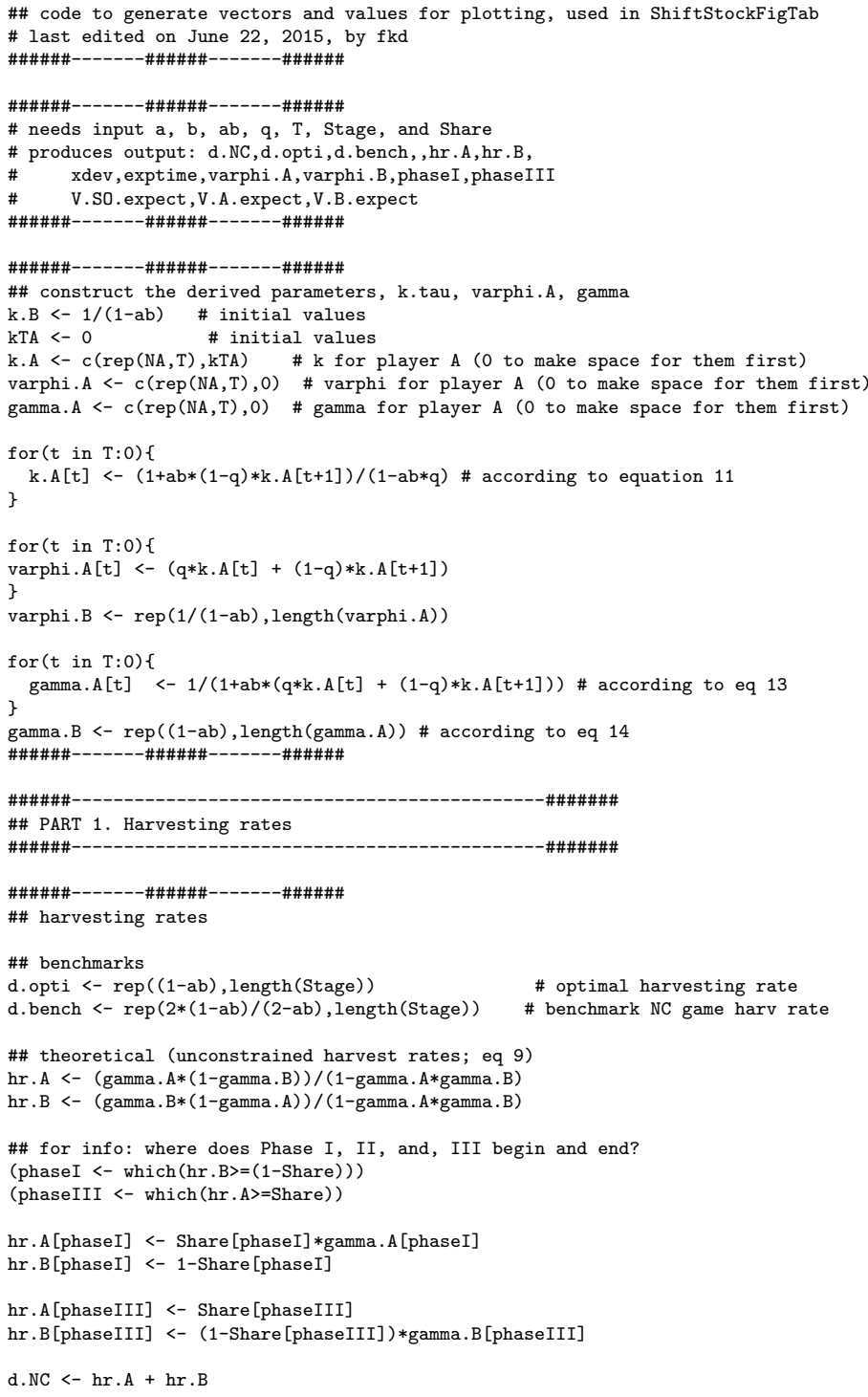




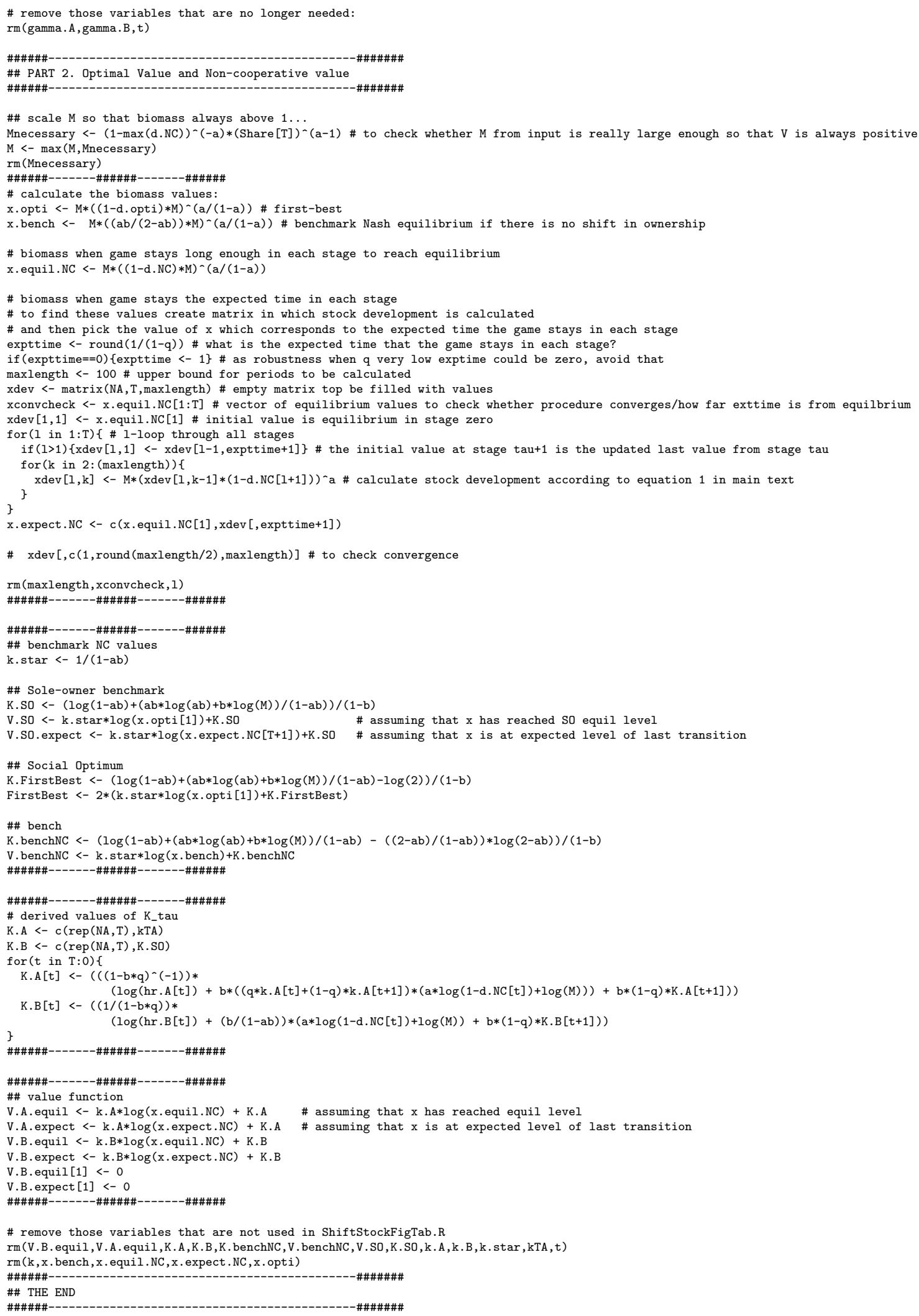

